\title{
Les enjeux de la traduction poétique Le cas du « Lac » de Lamartine
}

\author{
Par \\ Magdi Adli Ahmed ALI \\ Professeur adjoint \\ à la faculté de langues et de traduction \\ université d'Al-Azhar
}

2018 


\section{$\underline{\text { 0. Introduction }}$}

Poète, romancier et dramaturge français, Alphonse de Lamartine est l'une des grandes figures du romantisme en France au $19^{\text {ème }}$ siècle. Ce siècle a enrichi la littérature mondiale par de grands et immortels, romanciers et poètes, et par l'émergence des écoles et de différentes tendances littéraires, artistiques et intellectuelles. Son recueil «Les Méditations Poétiques », publié en 1820, a obtenu un grand succès fulgurant, et considéré comme le point de départ du Romantisme en France. Parmi ses différents poèmes, il nous a fallu trouver un poème qui puisse être adéquatement étudié. Ayant lu les différentes écritures sur ses poèmes, on voit qu'il serait enrichissant de s'intéresser au poème $\mathrm{du}$ « Lac » inspiré par la relation du poète avec une femme mariée et condamnée par la mort et connue sous le nom de Julie Charles. Triste et solitaire, le poète a écrit son célèbre poème " Le lac » pour exprimer sa détresse face à la perte de son amante. Souvent, il revient seul près de ce lac où il passait des instants heureux avec son amoureuse et réfléchissait sur la fuite du temps.

La présente recherche aborde les enjeux liés à la traduction poétique. En général, il existe des expressions dont le sens est équivoque pour les traducteurs, parce que la poésie renferme des images qu'il est difficile de cerner minutieusement le sens, cela nous justifie les différentes traductions pour un même poème. Aussi va-t-on essayer de souligner que : "Vu la grande richesse sémantique et des concepts évoqués dans la poésie, le traducteur se trouve face à une multitude de significations qu'il doit rendre dans la langue réceptrice'». Le lac a été traduit plusieurs fois en arabe : "presque vingt traductions différentes ${ }^{r}$ ». Les poètes et

') AMID, Abdallah, la fidélité en traduction double pour qu'elle soit belle et fidèle, Acte de Colloque, fac. Des sciences humaines, Al Azhar, le Caire, 2015, p.9 
les traducteurs arabes ont rivalisé dans le transfert de ce poème en arabe tels que : "Ahmed Hassan Al-Zayat [...], Mohamad Assad Wilaya [...],Ahmed Shawki [...], Ali Mahmoud Taha, Ibrahim Naji, Nicola Fiyad, Abi El-Fadl El-Walid, Al-Awadi AlWakil, El-Yas Abi Shabkah, [...], Dr Mohamed Mahdi Al-Basir, Abdul Razzaq Hamida, Mohamad Ghallab, Mohamad Mandor, Mohamad Ghenimi Helal, Ilya Hawi, Salah Ed-Dyn El-Monjed, Abd-Er-ahman Badawi etc." »

Le cadre de cette étude porte sur les langues française et arabe, et l'argumentaire se fonde sur le poème du «Lac » et quatre de ses différentes traductions, produites par Nicolas Fayad', Mohamed Mandour ${ }^{\circ}$ Idris El-Waghiche` et Mohamed ElSamahy .

Avant de traiter le sujet, il nous paraît essentiel de définir la poésie. Selon le Petit Robert, elle est un "art du langage, visant à exprimer ou à suggérer par le rythme (surtout le vers), l'harmonie et l'image ». De là, notons que la poésie est un genre littéraire singulier contenant de différentes caractéristiques telles que : "le rythme », "l'harmonie », et "l'image ». Il s'agit donc d'une créativité artistique, car : "Création et mystère forment le trésor de la poésie ».

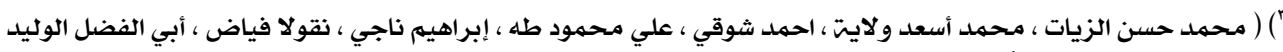

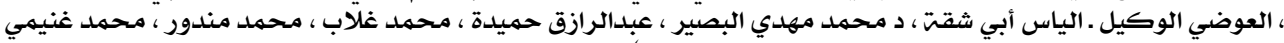

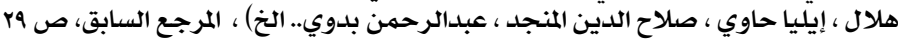

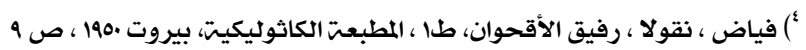

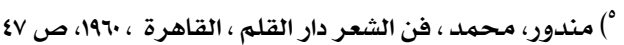

7) Poète, écrivain et professeur au Royaume du Maroc, Université Sidi Mohamed Ben Abdellah, Faculté des Lettres et des Sciences Humaines. Saïs -Fès.

http://www.ahewar.org/debat/show.art.asp?aid=502566

v) Poète marocain, http://www.matarmatar.net/threads/4931/

^) REY, A. et REY-DEBOVE J., Le Petit Robert, , Robert, Paris, 2001

`) PIERRE, J., Euvres II, texte établi et présenté par J Starobinski, Mercure de France 1987, p.1056. 
On se demande également si traduire la poésie est possible ou si les décalages existant entre les langues la rendent intraduisible? La poésie est en fait au cœur du débat sur la possibilité et l'impossibilité de la traduction. Des traductologues affirment qu'elle est intraduisible. Illustrant l'ampleur de cette tendance, on cite que "la poésie est par essence, nécessairement, intraduisible, sauf à vouloir, par souci d'information, en indiquer l'existence, et accepter la perte essentielle qui se produit, au passage d'une langue à l'autre "». Maurice Blanchot paraît rendre la traduction poétique impossible quand il écrit : "Le sens du poème est inséparable de tous les mots, de tous les mouvements, de tous les accents du poème. Il n'existe que dans cet ensemble et il disparaît dès qu'on cherche à le séparer de cette forme qu'il a reçue. Ce que le poème signifie coüncide exactement avec ce qu'il est" ». De même, Berman, souligne que "la poésie soit intraduisible, cela signifie deux choses : qu'elle ne peut pas être traduite, à cause de ce rapport infini qu'elle institue entre le "son » et le "sens 》, et qu'elle ne doit pas l'être, parce que son intraduisibilité [...] constitue sa vérité et sa valeur. Dire d'un poème qu'il est intraduisible, c'est au fond dire que c'est un "vrai » poème "». Ces citations sont un échantillon écrit sur l'impossibilité de traduire la poésie.

Pour nous, la traduction de la poésie n'est pas impossible mais difficile, ce qui pousse le traducteur à chercher des solutions créatrices et positives. Il est judicieux de rendre, dans la langue cible, un poème pouvant contenir les mêmes exigences et critères de qualité que la poésie lamartinienne. Le français et l'arabe sont deux langues dont le fonctionnement diffère. Elles ne se

1.) PENISSON, Pierre, «Le génie traducteur», in : M. Broda (éd.), La traduction-poésie, à Antoine

Berman, Presses universitaires de Strasbourg, Strasbourg, 1999, p. 136.

") GENETTE, G., La Littérature au second degré, in Palimpsestes. Seuil, Paris, 1982, p. 239.

") BERMAN, Antoine, «La traduction et la lettre ou L'auberge du lointain », Seuil, Paris, 1999, p. 60. 
correspondent pas, et il faut alors " être prêts à sacrifier certaines minuties formelles dans l'intérêt du contenu" ». Pour traduire, on peut sacrifier car le but de la traduction est «la captation du sens, c'est détacher celui-ci de sa lettre, de son corps mortel, de sa gangue terrestre. C'est saisir l'universel et laisser le particulier ${ }^{\prime \xi}{ }^{\prime}$. Les différences entre les langues existent, et le traducteur en somme "vit la différence des langues, toute traduction est fondée sur cette différence, tout en poursuivant, apparemment, le dessein pervers de la supprimer [...] A la vérité, la traduction n'est nullement destinée à faire disparaître la différence dont elle est au contraire le jeu : constamment elle y fait allusion, elle la dissimule, mais parfois en la révélant et en l'accentuant, elle est la vie même au cour de cette différence ${ }^{10} \gg$.

Le traducteur de la poésie rencontre souvent des difficultés provenant de la nécessité de rendre la valeur poétique du style imagé du texte source. L'image poétique contribue à concrétiser des idées abstraites. Cet article consiste à mettre en relief les caractéristiques spécifiques de ce type d'images, lors du transfert de la langue d'origine à la langue réceptrice, et donner des solutions appropriées au message original. Le traducteur doit tenter de reformuler le sens dans la langue du retour en faisant une économie de mots inutiles. "À valeur communicationnelle égale, la traduction la plus court sera la plus pertinente "». Il lit l'original avec «les yeux d'un traducteur " $"$ pour délimiter le procédé de transfert convenable.

ir) MESCHONNIC, Henri, Poétique du traduire, Verdier, Paris, 1999, p. 334.

") BERMAN, Antoine, «La traduction ....», Op. cit., p. 53.

10) BATTAGLIA, Anna, GARDES-TAMINE, J., « Traduire la poésie : du mot au texte », in : Synergies Italie, 2010, $\mathrm{n}^{\circ} 6$, p. 74

") VAN HOOF, Henri, « recherche d'un modèle d'analyse en traduction », s Méta, vol.16, 1971, p. 94

'v ) DELISLE, J., La traduction raisonnée, PUO, Canada, 2010, p. 129 
On espère que cette étude contribue à mettre en relief quelques points posant problème dans le transfert de l'expression imagée dans la langue d'arrivée. Cette recherche a pour principal objectif de reconnaître les décalages récurrents de traduction des textes poétiques. De là, on abordera le style lamartinien à partir de certaines figures de style qui confirment son habilité de s'en servir. On traitera les traits stylistiques et les traits poétiques du texte source "pour aboutir à un texte d'arrivée équivalent " $"$ ". La méthode suivie sera analytique et comparative pour illustrer le péril de poser un poème sous la pratique de la traduction, et savoir si le résultat obtenu est une traduction fidèle au poème original.

\section{Les traits stylistiques}

Les figures de style sont fréquemment évoquées comme étant caractéristiques de la poésie lamartinienne. Elles mettent en relief la beauté de son style. On pense particulièrement aux métaphores, périphrases, synecdoques, allégories, personnifications, répétitions, parallélismes, etc. qui sont généralement dérivées de la culture de la langue utilisée dans la formulation de la poésie, et là, réside le problème. GardesTamine affirme que la poésie n'est pas autre chose que l'utilisation systématique des images et qu' "à mesure que les caractéristiques formelles de la poésie se relâchent et disparaissent, elle [l'image] prend une part progressivement plus importante et devient peu à peu la caractéristique définitoire de la poésie, seul lien qui demeure entre le poème en prose et le poème en vers, seule marque qui oppose la poésie à tous les autres usages du langage ${ }^{\prime \prime} »$.

\subsection{La métaphore}

" ) OSEKI-DEPRE, Inès, Traduction \& poésie, Maisonneuve \& Larose, Paris, 2004, p.116.

${ }^{19}$ ) GARDES-TAMINE, J., MOLINO, Jean, Introduction à l'analyse de la poésie, 1982, p. 178 
C'est une comparaison sans l'outil de comparaison. L'outil qu'on utilise quand on fait la comparaison, on le lève et on tombe sur la métaphore qui est «une figure de style qui consiste à en une comparaison elliptique fondée sur l'analogie de deux objets, de deux notions, de deux situations représentant quelque caractère commun. Elle s'adresse à la fois à la raison et à l'imagination, et représente pour l'écrivain et le rédacteur une formidable économie de moyens. La métaphore dynamise un récit et rehausse son pouvoir évocateur par la production d'images mentales ${ }^{r} \gg$. Elle consistent alors à présenter une idée sous le signe d'une autre idée plus frappante ou plus commune, "qui d'ailleurs, ne tient à la première par aucun lien que celui d'une certaine conformité ou analogue ${ }^{r \prime} »$. Alors, c'est une figure de signification qui joue sur la relation entre les signes et la manifestation de la fonction symbolique de la langue. Elle consiste à exprimer une idée ou à évoquer un objet conformément à une façon particulière de concevoir et de représenter le réel. C'est une création de correspondances inédites dans la réalité, c'est le langage poétique par excellence. Ça veut dire la métaphore est très difficile à'utiliser dans la réalité.

Dans le poème, il existe plusieurs formes des métaphores. Elle est l'une des figures de style la plus fréquente et la plus expressive qu'on peut trouver dans une œuvre poétique. La réflexion sur le temps repose principalement sur l'usage de métaphores qui mettent en relief l'aspect fugitif du temps, qui s'écoule sans que l'homme qui le traverse puisse s'y arrêter :

Ainsi, toujours poussés vers de nouveaux rivages, (v.1)

Dans la nuit éternelle emportés sans retour, (v.2)

r.) DELISLE, J., La traduction raisonnée, Op. cit. p. 502

") FONTANIER, les figures du discours, Flammation, Paris, 1977, p. 99 
Ne pourrons-nous jamais sur l'océan des âges

(v.3)

Jeter l'ancre un seul jour? (v.4)

Le poème est basé sur une métaphore philosophique «l'océan des âges ». Là, le temps est comparé à un océan. Ils sont tous les deux si vaste qu'on n'en voit pas le bout. On peut dire également que le temps est aussi fluide que l'eau. Impossible de la saisir. "Les nouveaux rivages » représentent les jours qui se succèdent du coté de la vie. "La nuit éternelle », sans retour, représente bien sûr la mort. Ce poème nous présente ici une réflexion sur la vie et la mort.

La métaphore est riche en contrastes «toujours - jamais», "éternelle - un seul jour ». Le mouvement et la brièveté avec les verbes « emporter - pousser » s'opposent au désir de s'arrêter, de se reposer. "Jeter l'ancre », c'est la métaphore du port, du havre de paix auquel on aspire mais que la vie ne nous laisse aborder qu'à la fin du parcours.

Le poète semble s'adresser à nous directement « Ne pourrons-nous jamais ». Il inclut le lecteur et l'humanité entière dans cette question rhétorique. C'est-à-dire une question qui n'attend pas de réponse. Car la réponse est évidente. Non, en effet, il est impossible d'arrêter le temps. Ce premier quatrain illustre bien un discours philosophique.

Ce n'est pas l'originalité de l'idée qui va être marquante dans ce poème, mais plutôt la manière dont le poète va l'exprimer, d'une manière qui va rencontrer la sensibilité esthétique de son époque.

Pour rendre cette image en langue réceptrice, signalons que la saturation de l'esprit de deux cultures et de deux civilisations en question, la réincarnation de la personnalité du poète dans son poème, la connaissance de ses vers, l'extraction de 
son essence, la compréhension du vouloir dire avec précision, et la découverte des significations cachées se terminent inéluctablement par un transfert clair et précis du texte source.

\begin{tabular}{|c|c|}
\hline \multicolumn{2}{|c|}{$\begin{array}{l}\text { Ainsi, toujours poussés vers de nouveaux } \\
\text { rivages, (v.1) } \\
\text { Dans la nuit éternelle emportés sans retour, } \\
\text { (v.2) } \\
\text { Ne pourrons-nous jamais sur l'océan des âges } \\
\text { (v.3) } \\
\text { Jeter l'ancre un seul jour? (v.4) }\end{array}$} \\
\hline N. Fayad & 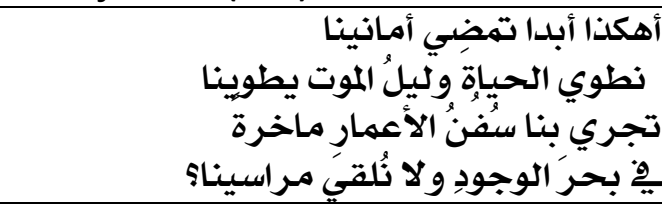 \\
\hline M. Mandour & 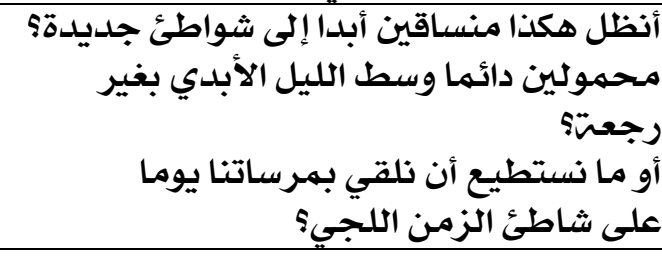 \\
\hline El-Waghiche & 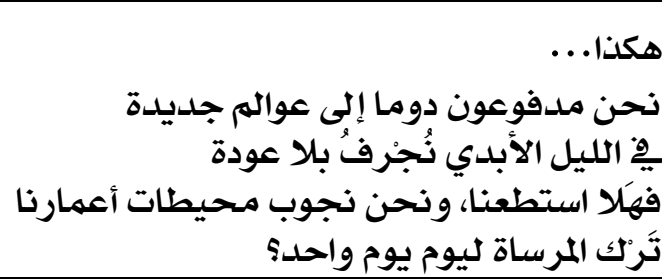 \\
\hline $\begin{array}{l}\text { M. El- } \\
\text { Samahy }\end{array}$ & 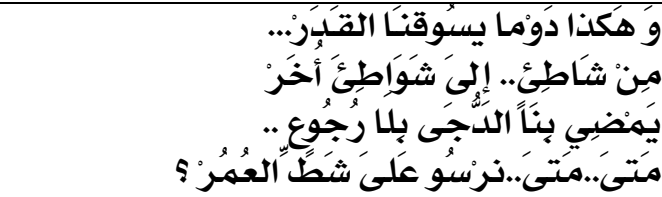 \\
\hline
\end{tabular}

Les utilisateurs de deux langues en question ne détiennent pas inévitablement la même sensibilité vis-à-vis des expressions métaphoriques, car en arabe "la pensée ne court pas sur les mêmes rails qu'en français " $"$. La traduction de Nicolas Fayad 
est une poésie verticale. Il ne cherchait que les termes appropriés à la rime et à la mesure. Il a eu recours à l'ajout injustifié pour garder la rime. "Trop souvent, la traduction rimée tourne alors au pastiche et son infériorité par rapport à l'original devient trop

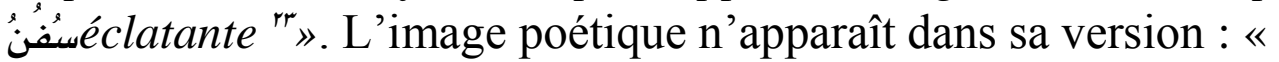
الأعمار ماخرة" Il a sacrifié l'exactitude au profit de la fluidité. Car chez lui : "Préserver la valeur esthétique des effets sonores, est la plus haute ambition dans la traduction de la poésie ${ }^{\mathrm{r \xi}} \gg$.

La traduction de M. Mandour est adéquate parce qu'il a rapporté tout ce qui est écrit dans la version française. Il a enrichi le texte avec le transfert de l'image métaphorique «l'océan des » qui résulte de sa شاطئ الزمن propre expérience. «Dans la traduction poétique, on est parfois en droit d'introduire des métaphores ou images exotiques, des mots étrangers dans la langue réceptrice pour provoquer des émotions ou picotements ${ }^{r^{\circ}} \gg$. Le bon traducteur doit chercher une solution qui conserve, en langue cible, les traits originaux du texte source et transmettre le message de départ en langue d'arrivée de façon à ce que le récepteur se trouve en train de lire une œuvre conçue dans sa propre langue.

La métaphore pourrait être littéralement traduisible. Telle la version de El-Waghiche. Une traduction littérale aboutit à la ». Sa traduction en prose محيطات أعمارناformulation suivante : « répond, de manière admise, à un désir de « fidélité ». Malgré son recours à la traduction rimée, El-Samahy a pu équitablement 》.شَطَِّ العُمُرْtransférer le message original : «

Prenons également cet exemple :

"r ) ELLRODT Robert, comment traduire la poésie, Op. cit. p. 71

$\left.{ }^{r}\right)$ RICOEUR, P., La Métaphore vive, Seuil, Paris, 1975, p. 265.

ro ) AMID, Abdallah, la fidélité en traduction double pour qu'elle soit belle et fidèle, Op. cit., p. 4 


\section{$\hat{O}$ temps, suspends ton vol! (v.21)}

Là, «le temps » est représenté par la métaphore de l'" oiseau - vol » qui nous montre que le temps s'écoule vite. Le comparé : le temps, le comparant : un oiseau (vol), le point de comparaison : la fuite. Nous pouvons littéralement traduire cette image parce que la traduction littérale nous offre une image poétique bien peinte dans le texte arabe :

\begin{tabular}{|c|c|}
\hline Fayad & يا دهر قَفن، فحرامُ أن تطيرُ بنا \\
\hline Mandour & أيها الزمن قف جريانك \\
\hline El-Waghiche & يا دهرَ مُهَها: لا تتعجَّل وانتظر قليلا \\
\hline El-Samahy & 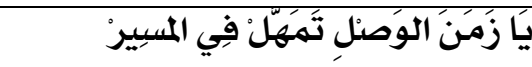 \\
\hline
\end{tabular}

Les traducteurs ont étonnamment donné un style imagé. C'est une traduction libre avec des différences stylistiques et métaphoriques. Chacun cherche une équivalence idéale. A cause de différentes structures linguistiques et culturelles de deux langues, Fayad semble traduire librement, favoriser l'expression française, et tenter d'ajouter des termes inexistant dans le poème » pour bien garder la rime en arabe. «La فحرامّ أن تطيرَ بنا Saurce 《 traduction entraîne nécessairement certains ajouts et certaines omissions d'information ${ }^{r \eta} \gg$. Pour ne pas tomber dans l'illusion, les autres traducteurs ont recours à reformuler les mots du texte source à la lumière de son interprétation contextuelle pour chercher dans la langue cible la bonne équivalence sémantique. Pourtant, cette image est adéquatement formée.

Quand une métaphore se prolonge sur un paragraphe, dans le discours, et quand elle se prolonge sur tout au long d'une 
strophe dans un poème, on appelle ça la métaphore filée ${ }^{\mathrm{rv}}$. Voilà un exemple tiré du Lac:

et vous, heures propices, (v.21)

Suspendez votre cours! (v. 22)

Ici, « le temps » est représenté par la métaphore de "l'eau » qui est filée tout au long du poème. Le comparé : le temps (heures), le comparant : un cours d'eau, le point de comparaison : le temps passe, «coule » comme de l'eau. Nous observons la métaphore du temps assimilé à l'eau. C'est l'idée de «l'eau » qui coule comme «le temps » qui s'écoule. Pour rendre cette image métaphorique, les traducteurs ont recours à la traduction libre :

\begin{tabular}{|c|c|}
\hline Mandour & وأنتي أيتها الساعات السعيدة قفي \\
\hline El-Waghiche & 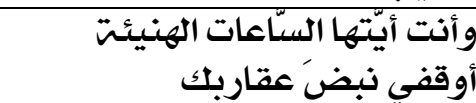 \\
\hline El-Samahy & 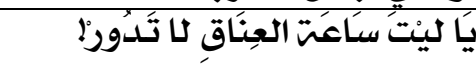 \\
\hline
\end{tabular}

Traduisant d'une langue à l'autre, plusieurs défis pourraient rencontrer le traducteur, surtout, lorsqu'il rend la métaphore. En effet, il doit essayer de rendre le contenu et le vouloir dire de l'image métaphorique. Il doit également conserver autant que possible les traits formels de la figure.

En ce qui concerne la raison qui a poussé Mandour et ElWaghiche à opter pour une traduction en prose, c'est la crainte de dénaturer et d'altérer le poème original. Les deux traducteurs ont probablement traduit le poème en croyant qu'ils ne pourraient jamais arriver à une pareille création poétique. ElSamahy s'intéresse au vouloir dire et maintient la lettre et le sens

${ }^{r v}$ ) C'est une métaphore développée par un champ lexical qui traverse un texte, un poème, un paragraphe, une strophe. C'est un réseau complexe de métaphores. 
de l'original ayant recours à une «singerie rimée ${ }^{\Uparrow t} »$. Malgré les différentes versions, les traducteurs ont pu produire la même impression que le message source. Ces versions sont pertinemment fidèles.

Au préalable, le traducteur doit chercher à présenter cette figure de rhétorique dans sa traduction chaque fois qu'il le peut. En présence une métaphore à traduire, trois possibilités s'offrent à lui en général :

a) la traduction littérale. Mais, parfois «la langue d'arrivée ne permet pas de traduire la métaphore littéralement ${ }^{\mathrm{rq}} \gg$.

b) l'emploi d'une autre métaphore de sens proche ou équivalent en langue d'arrivée. "Les métaphores, d'une langue à l'autre, se correspondent absolument ou à peu près "». C'est souvent ce qui arrive lorsque les deux langues en présence ont des traditions communes.

c) si les deux précédentes solutions sont inapplicables, le traducteur se contente de rendre l'idée sous-jacente aux images du texte de départ. "On essaiera de trouver une équivalence et, si ce n'est pas possible, on s'attachera à traduire l'idée "'».

En outre, le traducteur pourrait volontairement introduire ou créer une métaphore dans le texte d'arrivée, même s'il n'existe pas dans le texte de départ, "il n'est pas au contraire au principe de la traduction d'introduire ponctuellement dans le texte d'arrivée une métaphore usée ou vive, même si cette figure

‘` ) BAUDELAIRE, Charles. Histoires grotesques et sérieuses. Michel Lévy frères, Paris, 1865, p. 336

${ }^{r}$ ) VINAY, DARBELNET, Stylistique comparée du français et de l'anglais, Dédier, Paris, 1999, p. 199

r.) Ibidem

${ }^{r 1}$ Ibid., p. 200 
de rhétorique est absente du texte de départ "r». De plus, le traducteur pourrait rendre un segment source au moyen d'un idiotisme non suggéré par la formulation du texte original. Ce qu'on appelle un "renforcement du caractère idiomatique $d u$ texte d'arrivée ${ }^{r \prime}$.

\subsection{La personnification}

Elle consiste à « attribuer à des choses inanimées ou à des entités abstraites des comportements, des sentiments ou des attitudes caractéristiques des êtres humains ${ }^{\prime \xi} \gg$. Elle fait d'un être inanimé ou d'une idée abstraite un personnage réel, doué de vie et de sentiments. Elle crée l'impression que les choses vivent et pensent, que les idées ont une existence autonome, indépendamment des hommes ${ }^{r_{0}}$. Le «lac » est riche en personnification.

La nature est personnifiée. Le poète la considère comme une confidente qui lui exprime ses sentiments mélancoliques.

Ôlac! Rochers muets! Grottes! Forêt obscure! »(v.46).

Il s'adresse à la nature, « $a u l a c$ » et à ses attributs «Rochers muets! Grottes! Forêt obscure! », pour lui exprimer ses tristes sentiments et lui raconter qu'il s'ennuie des moments passés avec l'être aimé. Le poète interpelle les éléments de la nature comme une apostrophe à un ami. En tant que cette image existe en arabe, les traducteurs l'ont rendue par son équivalent. Les traducteurs choisissent la traduction littérale pour rendre cette figure en arabe :

") DELISLE, J., La traduction raisonnée, Op. cit. p. 510

ri) Ibidem

${ }^{r s}$ Ibid., p.28

ro ) http://www.espacefrancais.com/la-versification/ consulté le 05/01/2018. 
Les enjeux de la traduction poétique Le cas du « Lac » de Lamartine

\begin{tabular}{|c|c|}
\hline Mandour & 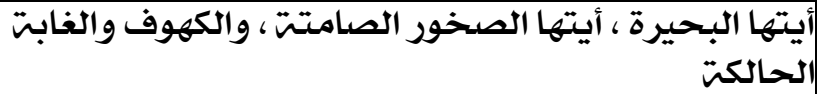 \\
\hline El-Waghiche & 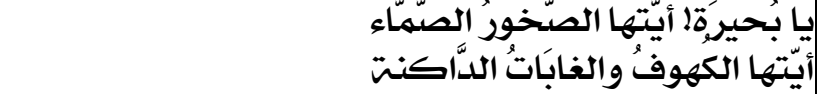 \\
\hline
\end{tabular}

Là, la littéralité se substitue à l'équivalence adéquate et l'image est bien peinte en arabe. Les traducteurs s'adressent au

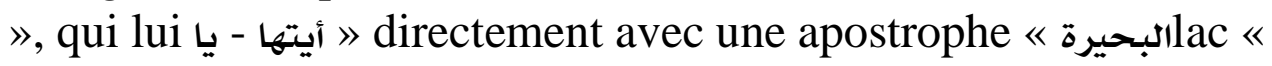
confère des caractéristiques humaines.

Pour le poète, la nature représente "un lieu de repos approprié à la contemplation et un lieu de refuge " $"$. Elle est considérée comme une confidente fidèle et une amie sensible du poète. Elle s'anime et est humanisée par le poète qui lui donne des qualités humaines : «Regarde! », «t'en souvient-il ?». En fait, «le lac » est l'image la plus répandue et la plus expressive dans ce poème. Il est personnifié par le poète qui s'adresse à lui en le tutoyant «tu la vis s'asseoir»:

Ô lac ! L'année à peine a fini sa carrière ( $v$.

7)

Et près des flots chéris qu'elle devait revoir, (v. 8)

Regarde! Je viens seul m'asseoir sur cette pierre (v. 9)

Où tu la vis s'asseoir! (v. 10)

Un soir, t'en souvient-il ? Nous voguions en silence ; (v. 13)

Là, on a quelque chose qui existe réellement, qu'on voit, qu'on regarde, c'est «le lac » qui vit, et qui a des yeux, c'est un être animé. On lui confère, avec un verbe « regarder », des caractéristiques humaines. Et là, c'est une personnification, parce

ๆ) ONDRUSKOVA, Dana, la nature dans les recueils les méditations poétiques et les destinées de Lamartine et Vigny, Thèse de baccalauréat, Université de Masaryk À Brno, 2011 p. 15

Par / Magdi Adli Ahmed ALI 
que ici on ne parle pas quelque chose d'abstrait, on parle quelque chose de concret. Un lac, normalement, ne peut pas regarder. Mais quand il " regarde », c'était comme un être humain, un homme ou une femme ayant des yeux pour regarder. Le poète vise à humaniser "le lac ». Il devient l'ami fidèle et sincère, le copain sensible et le confident du poète qui lui fait part de sa solitude. Le poète revient souvent au lac pour lui raconter sa bien-aimée, ses plaintes langoureuses et ses souffrances. Il demande au lac de lui rendre les souvenirs des moments merveilleux passés avec son amante.

La personnification du «lac » est présentée dans le dialogue du poète avec «le lac». Il s'adresse à lui comme à un interlocuteur «tu la vis s'asseoir », "Un soir, t'en souvient-il ? ». Le poète " donne la vie » à la nature «tu - lac». Ce n'est pas une illusion ou une hallucination. Le poète a besoin d'humaniser un monde tout à fait indifférent. "Le lac » garde le souvenir. C'est le poète qui lui donne cette capacité. C'est un dialogue fantastique et imaginaire pour faire face au sentiment de solitude et de tristesse, aux souvenirs immortels et à l'amour éphémère. Mais le lac ne dit rien, et le poète est toujours seul. Il cherche à retrouver, près du lac, les moments heureux de son amour. Pourtant, du fait de la littéralité strictement faite par les traducteurs, cette image est pertinemment formée en arabe.

\begin{tabular}{|c|c|}
\hline Mandour & 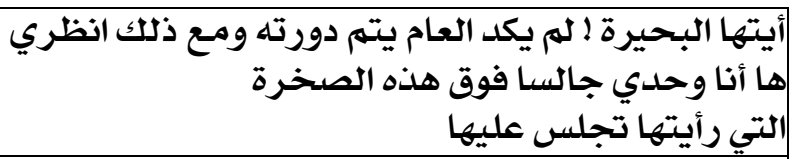 \\
\hline El-Waghiche & 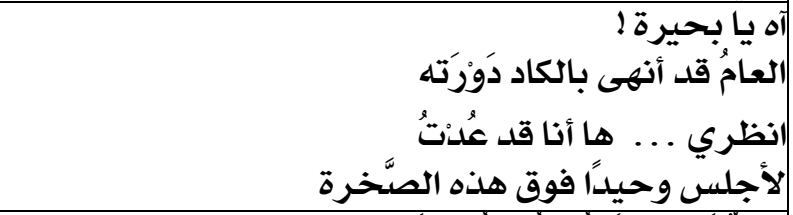 \\
\hline El-Samahy & 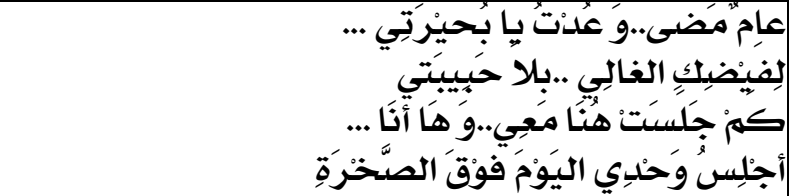 \\
\hline
\end{tabular}


La personnification est représentée dans les deux textes (source »dont أيتها البحيرة - يا بحيرة - يا بُحيُرَتي اiet cible) par le terme « l'équivalent est « $\hat{O} l a c »$. Comme le poète, les traducteurs » directement avec une apostrophe البحيرة' البحيرة'adressent au lac «

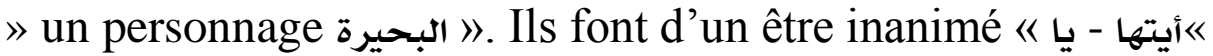
réel, doué de vie. Ils humanisent un monde tout à fait indifférent ». Alors, la traduction نظريs'adressant à lui à l'impératif « littérale de cette image est indispensable afin d'atteindre le vouloir dire du poète.

Le temps est ainsi l'une des images lamartiniennes. Le poète s'adresse directement au temps. Il transforme l'inanimé en animé. Il nous donne une image personnifiée afin d'immortaliser ses souvenirs. Personnification du temps telle que : «Ô temps, suspends ton vol! et vous, heures

propices, (v.21)

Suspendez votre cours! (v.22)

Laissez-nous savourer les rapides délices (v.23)

Des plus beaux de nos jours! (v.24)

Tout au long du poème, le temps est personnifié. Le poète s'adresse directement au temps. Celui-ci est présenté métaphoriquement comme un oiseau grâce au nom « vol » puis comme une rivière grâce au nom " cours ». Le verbe "suspendre » répété deux fois lui renferme son caractère humain. Il se plaint du temps qui passe trop rapidement. Il implore le temps d'arrêter, de ne pas couler « $\hat{O}$ temps, suspends ton vol! », ou de reculer pour revivre à nouveau " Des plus beaux de nos jours !». La traduction littérale nous offre une image poétique bien peinte en arabe :

\begin{tabular}{|c|c|}
\hline Mandour & وأينت أزتها الساعات جريانكيدة قفي انسيابك \\
\hline El-Waghiche & 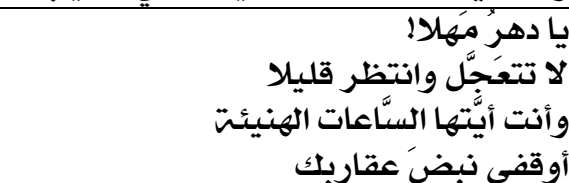 \\
\hline El-Samahy & 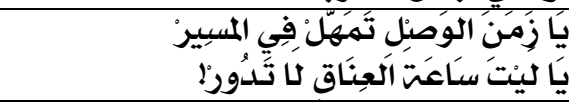 \\
\hline
\end{tabular}


Voyons également ces personnifications :

Le temps m'échappe et fuit ; (v.30)

\begin{tabular}{|c|c|}
\hline Mandour & فالزمن يفلت ويهرب \\
\hline El-Waghiche & الزمن ينفلتُ منّي، ويهضي: \\
\hline El-Samahy & 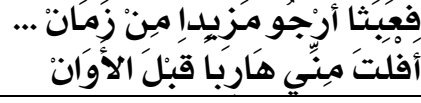 \\
\hline
\end{tabular}

Il coule et nous passons! (v.36)

\begin{tabular}{|c|c|}
\hline Mandour & إنه ينساب ونتسـاب معهد \\
\hline El-Waghiche & يُمضي ونحنُ مَاضون مُعَها. \\
\hline El-Samahy & هَذًَا يَسبيرِ - رَاحلا -وَ ذَا يَسبيلن \\
\hline
\end{tabular}

Temps jaloux, se peut-il que ces moments d'ivresse

(v. 37)

\begin{tabular}{|c|c|}
\hline Mandour & هلي يجوز الزمن الغيور \\
\hline El-Waghiche & 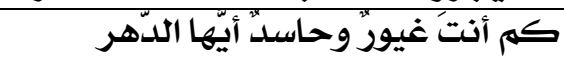 \\
\hline El-Samahy & 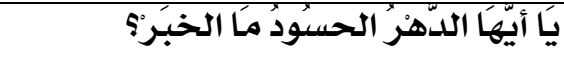 \\
\hline
\end{tabular}

Le temps est présenté comme un être humain qui "s'échappe et fuit». Il est également présenté comme un être vivant qui est « jaloux » où cet adjectif renforce la personnification. Les verbes d'actions «échappe » et «fuit » renforcent son caractère humain. Le poète exprime ses sentiments douloureux du temps qui passe. Cependant, il ne peut jamais contrôler le temps. Il ne peut pas échapper au temps qui s'enfuit : «Le temps m'échappe et fuit ». Les traducteurs ont La excellemment imité l'image du temps personnifié en arabe. traduction revêtue typiquement de la même image personnifiée serait plus compréhensible pour tous les récepteurs du message de la langue d'arrivée. 


\subsection{La périphrase}

La périphrase consiste à utiliser plusieurs termes pour indiquer un seul mot. C'est " une suite de mots qui exprime ce $q u$ 'il aurait été possible de dire en un seul mot ${ }^{r v}{ }^{»}$. Elle consiste à " exprimer une idée d'une manière détournée, étendue et ordinairement fastueuse, une pensée qui pourrait être rendue d'une manière directe, et en même temps plus simple et plus courte $^{r \wedge} \gg$. Alors, il s'agit de substituer une série de plusieurs termes à un mot ou à un groupe de mots, plus restreint, capable de désigner la même notion.

Dans l'astre au front d'argent qui blanchit ta surface ( $v$.

De ses molles clartés! (v. 60)

Là, le poète attire l'attention des lecteurs vers l'eau du lac ondulée doucement par le vent reflétant la lune sur la surface. Quand on dit «l'astre au front d'argent», on est en train de périphraser pour souligner la solennité de la scène. Cet astre a de «molles clartés» parce que sa lumière en est dispersée. En fait, le poète a recours à l'expression de "L'astre au front d'argent » pour désigner «la lune ». C'est à dire le fait de désigner un mot par un groupe de mots qui en décrit une caractéristique.

Le but est de créer une attente, un mystère ou attirer l'attention sur les qualités d'un objet. Il faut vraiment être quelqu'un de bien cultiver pour comprendre parfois la périphrase. Ce qui est beau dans les figures de style c'est qu'elles nous invitent à bien réfléchir, et parfois, elles nous frappent par l'intensité de l'expressivité. Pour rendre cette image en arabe, il faut d'abord signaler que nombreux traducteurs achoppent sur les messages métaphoriques, car la traduction exige une sensibilité linguistique, une création imaginaire, un logique et un bon goût.

${ }^{r v}$ ) DELISLE, J., La traduction raisonnée, Op. cit., p. 505

${ }^{r \wedge}$ ) FONTANIER, les figures du discours, Flammation, Paris, 1977, p. 361

Par / Magdi Adli Ahmed ALI 


\begin{tabular}{|c|c|}
\hline$\overline{\text { Fayad }}$ & 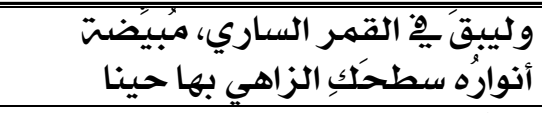 \\
\hline Mandour & 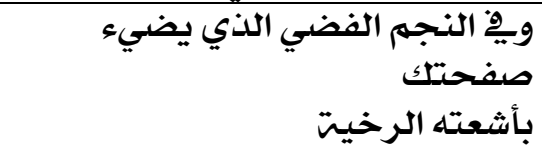 \\
\hline El-Waghiche & 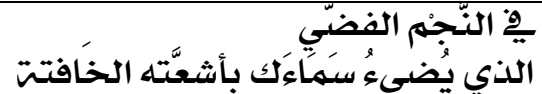 \\
\hline El-Samahy & 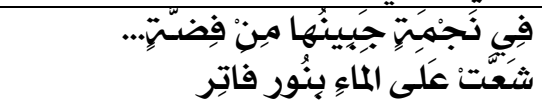 \\
\hline
\end{tabular}

Ce sont de différentes traductions d'un passage du poème du lac. Chaque traduction se distingue de l'autre en termes de sens, de pensée, de capacité à se conformer, de caractère inhérent de la connaissance du sujet et de connaissance de la langue de partir et celle de retour. Dans l'expression «l'astre au front d'argent» réside une image périphrastique. Fayad a rendu cette expression métaphorique par «son sens propre » un terme ». C'est-à-dire une suite القمرsimple désignant la même réalité « de mots rendus par un seul mot. Cette image est adéquatement formée en arabe.

Quant à la traduction d'El-Samahy, elle est inappropriée, avec un savoir-faire clair. Dû à une interprétation fautive, il traduit la périphrase «l'astre au front d'argent», par « من فِضَّنَّ faite par El-Samahy, cette image n'est pas pertinemment formée en arabe. Il n'arrive pas à déceler le vouloir dire du poète. Sa traduction perd le bon sens de l'origine où il a été forcé à cause de la rime ou de la nécessité poétique à l'utilisation involontairement du faux-sens.

La traduction de Mandour et celle d'El-Waghiche sont similaires. Ils ont rendu l'expression périphrastique par son ». Ils nous donnent une traduction imagée النَّجم الفضّئquivalent « et périphrastique. Leur traduction nous offre une image bien 
peinte en arabe, une traduction pertinente et adéquate, car "désigne «la lune ». Signalons alors النَّجمْ الفضّي اlexpression « que les versions de Fayad, de Mandour et d'El-Waghiche sont généralement plus fidèles et plus proches du modèle français, car "Ce qui importe à la traduction, c'est la fidélité au vouloir dire de l'auteur ${ }^{r q} \gg$.

Un autre exemple :

Le flot fut attentif, et la voix qui m'est chère

(v. 19)

Laissa tomber ces mots : (v. 20)

Là, la femme est nommée et présentée à travers une périphrase relative "qui m'est chère ». Le poète attire l'attention des lecteurs vers la bien-aimée qui est nommée et présentée à travers la périphrase. C'est un ton pathétique (lamentation). L'amante se lamente sur ce temps qui emporte cruellement les « rapides délices ». En fait, le poète a recours à l'expression «qui m'est chère» pour désigner «son amante ». Vu la littéralité strictement faite par les traducteurs, cette image est pertinemment formée en arabe.

\begin{tabular}{|c|c|}
\hline N. Fayad & 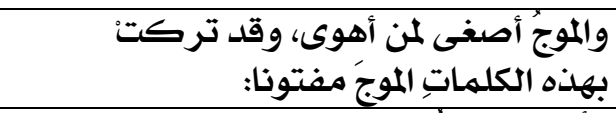 \\
\hline M. Mandour & 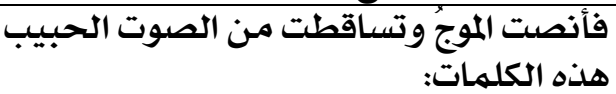 \\
\hline El-Wo & 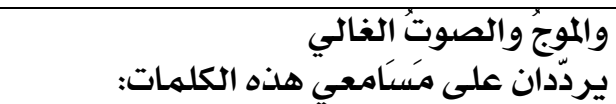 \\
\hline El-S & 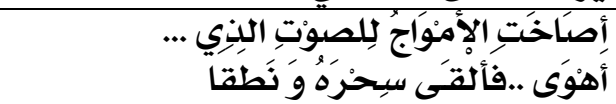 \\
\hline
\end{tabular}

${ }^{\text {ra }) ~ S E L E S K O V I T C H ~ D ., ~ \& ~ L E D E R E R ~ M ., ~ I n t e r p r e ́ t e r ~ p o u r ~ t r a d u i r e . ~ D i d i e r, ~ P a r i s, ~ 2001, ~}$ $\mathrm{P} 23$ 
Il y a une image périphrastique très forte dans ces passages. La traduction littérale nous offre une image poétique bien peinte en arabe. Elle ne conduit pas à dénaturer le sens.

\subsection{La synecdoque}

C'est « une figure de signification ou trope qui joue sur les relations de contiguïté entre objets (individus, événement) existant dans le monde et qui remplace le nom d'un des deux objets par celui de l'autre. Les deux objets ne sont pas indépendants l'un de l'autre, et sont liés par un lien de types définitionnel :- définition par le genre donnant lieu à la synecdoque de l'espèce (le nom de l'espèce est pris pour celui du genre) ou du gendre (le nom du genre est pris pour celui de l'espèce) - définition par énumération des parties donnant lieu à la synecdoque de la partie pour le tout" ».

C'est une image fondée sur une contiguïté logique. Elle exprime par une de ses parties une relation d'inclusion entre substituant et substitué. Une synecdoque consiste à parler de la partie pour le tout ou du tout pour la partie. Quand on parle « d'inclusion » ça veut dire qu'une partie qui est incluse dans un tout, dans un ensemble.

Ainsi le vent jetait l'écume de tes ondes (v.11) Sur ses pieds adorés. (v.12).

La femme aimée est évoquée par l'une de ses parties. Elle est alors idéalisée par La synecdoque. Le substituant est une partie du substitué. Les «pieds adorés » ne sont qu'une partie d'une amante. Donc, il y a une inclusion, on parle seulement d'une partie pour présenter le tout, l'ensemble. La traduction

") GARDES-TAMINE, J., HUBERT. M. C., Dictionnaire de critique littéraire, A. Colin, Paris, 2002, p. 210 
littérale nous offre une image poétique appropriée et systématique en arabe :

\begin{tabular}{|c|c|}
\hline N. Fayad & 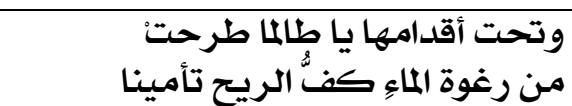 \\
\hline M. Mandour & وهوق قدميها المعبودياح تلقين بزبلد أمواجلك \\
\hline El-Waghiche & والذَّبيَّتين تلقي بزبـ أمواجلك على رجليها \\
\hline El-Samahy & 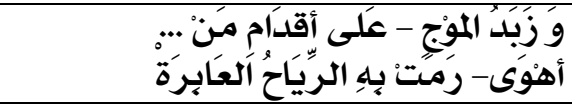 \\
\hline
\end{tabular}

Ce n'est pas suffisant de rendre le sens, mais il faut également traduire le style ou l'esthétique du poète et porter sur les images riches d'impressions et de suggestions. Les traducteurs ont remarquablement saisi l'idée poétique dans sa pureté originelle. Ils ont rendu de façon cohérente la synecdoque « ses pieds » par ». La قدامها - قدميها - رجليها - أَقَدَام Son équivalent en arabe 《 littéralité a conduit à une image adéquatement formée.

Un autre exemple :

Le flot fut attentif, et la voix qui m'est chère (v. 19)

Laissa tomber ces mots : (v. 20)

La personne aimée est désignée uniquement par sa voix, "la voix qui m'est chère ». C'est une synecdoque. La jeune fille n'est pas décrite, elle reste un mystère, évanescente. C'est une évocation d'une femme aimée. La synecdoque idéalise la femme amoureuse. Elle met en plus en valeur la femme. On parle la femme aimée qui est désigné ici par « la voix ».

\begin{tabular}{|c|c|}
\hline N. Fayad & 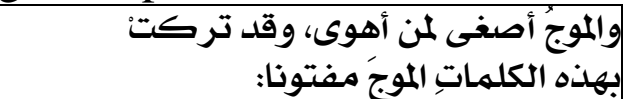 \\
\hline M. Mandour & هذأنص الكلمات الموج وتساقطت من الصوت الحبيب \\
\hline El-Waghiche & 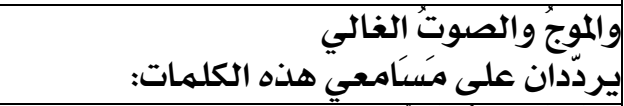 \\
\hline El-Samahy & 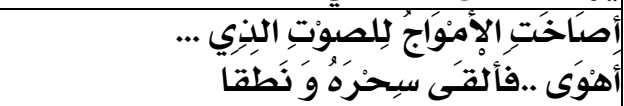 \\
\hline
\end{tabular}


«Le traducteur, tantôt lecteur pour comprendre, tantôt écrivain pour faire comprendre le vouloir dire initial, sait fort bien qu'il ne traduit pas une langue en une autre mais qu'il comprend une parole et qu'il la transmet à son tour en l'exprimant de manière qu'elle soit comprise $e^{\prime \prime} »$. Fayad a bien saisi l'idée originelle, mais il n'a pas gardé l'image poétique. En contrepartie, la traduction littérale de cette image est indispensable afin d'atteindre le vouloir dire du poète. Mandour, El-Waghiche et El-Samahy ont littéralement rendu l'image originelle «la voix » par son image équivalente en arabe

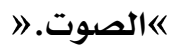

\subsection{L'allégorie}

Nous parlons d'allégorie lorsque "la métaphore filée évoque un sens caché sous le sens littéral "r»». Elle consiste à représenter une idée abstraite et morale par une image concrète.

«Ô temps, suspends ton vol! et vous, heures propices, (v.21)

Suspendez votre cours! (v.22)

$\therefore(v .25)$

Assez de malheureux ici-bas vous implorent

Coulez, coulez pour eux ; (v.26)

Là, l'allégorie, "temps - oiseau » et «temps - océan », a une importance singulière. C'est la représentation concrète d'une idée abstraite. Le «temps » est quelque chose d'abstrait, et n'est pas concrète. Le poète le fait ressembler à un «oiseau » qui vole : «Ô temps! Suspends ton vol », c'est un ordre adressé au

¿) SELESKOVITCH D., \& LEDERER M., Interpréter pour traduire, Didier, Paris, 2001, p. 19

«r ) DELISLE, J., La traduction raisonnée, Op. cit., p. 505 
«temps » comme à un « oiseau » pour suspendre son vol et se reposer. Il le compare à quelque chose de concret.

De plus, le poète demande au «temps » de dépêcher dans les instants difficiles comme l'océan face au danger « coulez, coulez », et de suspendre sa course pour perpétuer les instants de bonheurs «Suspendez votre cours ». Le vers «Assez de malheureux ici-bas vous implorent » convient à cette demande d'accélérer le temps afin de soulager les moments difficiles et les souffrances ressentis. Le temps est une notion abstraite bien sûr mais quand il est lié à «suspends ton vol» et à " suspendez votre cours », on lui confère quelque chose de concret, comme si le temps avait des ailes qui le font rendre voler et suspendre son vol.

Les deux langues n'ont pas de traditions communes. Elles sont tout à fait différentes. Cette différence est perceptible sur les plans lexical, syntaxique et stylistique. Le français préfère quelquefois insister sur l'abstrait pour représenter un objet concret.

Nombreux termes et expressions français recouvrent une conception de l'abstraction généralisée qui rend leur traduction très délicate en arabe. L'arabe pourrait rendre ces termes et ces expressions, soit par leur équivalent, soit par des termes concrets.

\begin{tabular}{|c|c|}
\hline Mandour & 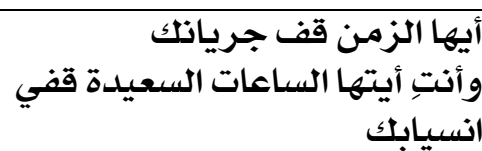 \\
\hline El-Waghiche & 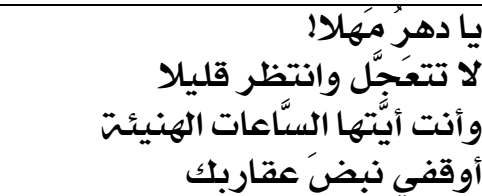 \\
\hline El-Samahy & 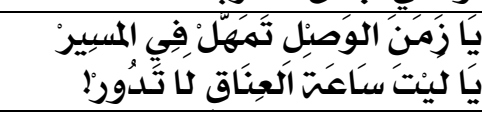 \\
\hline
\end{tabular}


ou الدهر - الزمن (المحيطL'allégorie, (

), permet de rendre plus sensibles ou plus frappantes les العصفور( notions abstraites. Les traducteurs ont recours à la traduction littérale pour adéquatement transférer cette image en arabe.

\subsection{L'antithèse}

C'est une figure qui consiste à rapprocher deux unités aux significations opposées ${ }^{\text {sr }}$. Elle consiste alors à mettre en opposition des termes désignant des réalités contradictoires. C'est -à- dire un placement dans un même énoncé deux notions opposées. Mais il faut signaler qu'il $\mathrm{n}$ y a pas de relation logique et objectif entre les deux énoncés. Prenons cet exemple ayant deux termes de sens antithétiques. L'image d'antithèse est évidemment établie dans cet énoncé.

Le temps m'échappe et fuit ; (v.32)

Je dis à cette nuit : Sois plus lente; et l'aurore (v.33)

Va dissiper la nuit ». (v.34)

Ces vers sont construits sur un sens contradictoire entre " échappe et fuit » et "Sois plus lente ». Donc, toujours c'est une opposition entre deux énoncés qui n'expriment pas la même réalité, où il n'existe pas de lien logique entre les deux termes sur le plan sémantique. Etant donné qu'elle existe en langue de retour, cette image antithétique a littéralement été bien rendue :

\begin{tabular}{|c|c|}
\hline M. Mandour & |وأقول لهنّا الليل ويهربل \\
\hline El-Waghiche & 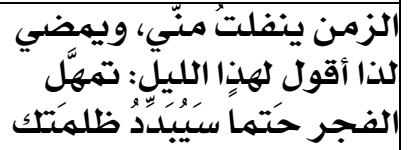 \\
\hline
\end{tabular}

«r ) GARDES-TAMINE J. \& HUBERT M. C., dictionnaire de critique littéraire, Op. cit. p. 16 
- échappe et يفلت ويهربLà, il y a deux mots antithétiques « - Sois plus lente ». En fait, l'antithèse est une تمfuit » et « forme éloquente indiquant normalement l'opposition de deux réalités contradictoires en arabe.

L'antithèse est également plus claire entre les verbes « donner » et « effacer » dans ces vers :

Ce temps qui les donna, ce temps qui les efface, (v. 43) Ne nous les rendra plus? (v. 44)

La construction parallèle du premier vers souligne l'antithèse. Ce vers est composé de deux hémistiches parallèles opposés à la fin à des verbes antithétiques, ainsi à de différents temps, passé « donna », présent « efface ». Signalons que "L'antithèse "ce temps qui les donna, ce temps qui les efface" suggère également la fugacité des moments de bonheur, qui disparaissent aussi vite qu'ils ont éclos "». La traduction littérale de cette image est indispensable afin d'atteindre le vouloir dire du poète.

\begin{tabular}{|c|c|}
\hline M. Mandour & وهن وهاها الزمن الذي منحها، والذي \\
\hline El-Waghiche & 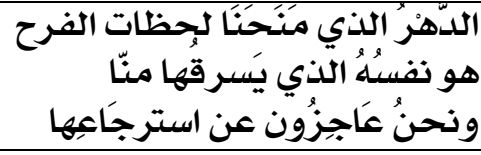 \\
\hline
\end{tabular}

منحها - مَنَحَنَا etes deux notions antithétiques « يسرقُها ئ employées par les traducteurs forment clairement cette figure de style en arabe. 
Le poète a énormément recours aux images antithétiques pour exprimer son génie et frapper l'imaginaire du lecteur. L'opposition est encore plus explicite dans ce vers :

Qu'il soit dans ton repos, qu'il soit dans tes orages, (v.49)

Les deux hémistiches sont construits de la même façon pour mieux opposer à la fin des mots antithétiques « repos » et « orages ». Voyons alors la traduction de cette image :

\begin{tabular}{|c|c|}
\hline M. Mandour & ويٌْ لحظات هدو ئك أو صخبك \\
\hline El-Waghiche & عِِ سُكونك أو صَخَبَك \\
\hline
\end{tabular}

Il y a une image antithétique très forte dans ce passage. La traduction de l'antithèse est marquée par l'emploi de deux termes contradictoires. Littéralement, cette image a été bien formée en arabe. La figure de l'antithèse est tellement frappante car elle attire notre attention à découvrir l'originalité du texte poétique. Le poète y a fréquemment recours pour exprimer son génie. Signalons alors que les figures de style ont toujours comme but le fait de frapper l'imaginaire, d'attirer le lecteur pour qu'il s'intéresse un style précis.

\subsection{La répétition}

On entend par répétition «la reprise d'un mot ou d'une structure syntaxique à proximité de la première occurrence ${ }^{\text {so }}$. C'est le retour de la même idée, du même mot ou groupe de mots. Par ailleurs, les répétitions sont très fréquentes dans le poème du «Lac». Elles jouent un rôle tant sur le plan rythmique que sur le plan sonore. Elles créent également une forme de refrain qui structure le poème. 
Ex: Dans les bruits de tes bords par tes bords répétés, (v.58)

On souligne la répétition du mot «bords». C'est une répétition évocatrice de l'action et du bruit qui battent les rochers du rivage et sont renvoyées par ces mêmes rochers et par l'écho". Voyons alors la traduction :

\begin{tabular}{|c|c|}
\hline M. Mandour & وبِ النغمات التي ترددها شطآنك \\
\hline El-W & \\
\hline
\end{tabular}

L'abus de termes rattachés les uns aux autres par la préposition «par» relève du style maladroit et nous donne un exemple de mauvaises répétitions. Il est judicieux de signaler que : "il existe un savoir-faire de la répétition. Car il y a, bien sûr, des répétitions mauvaises, maladroites. La règle: si on répète un mot c'est parce que celui-ci est important, parce qu'on veut faire retentir, dans l'espace d'un paragraphe, d'une page, sa sonorité ainsi que sa signification ${ }^{\langle v}{ }$. Les traducteurs ont alors eu recours à éviter les répétitions non souhaitables. Car l'arabe n'épreuve pas la même répugnance que le français à l'égard de la répétition.

De plus, la répétition pourrait créer un fait d'insistance valorisée et un rythme musical.

Ex : Coulez, coulez pour eux ; (v. 26)

La voix de la personne aimée est prolongée et dédoublée avec des effets d'écho «coulez, coulez ». La parole est mise en scène de façon spectaculaire. Le verbe «coulez, coulez » est repris plusieurs fois sans modification. La répétition du verbe scande le rythme et rend la supplique plus pressante. Rendre la

") https://www.comptoirlitteraire.com/docs/465-lamartine. Consulté le 11/11/2017

sv ) KUNDERA, Milan, Les testaments trahis, Gallimard, Paris, 1993, p. 138 
répétition en arabe est parfois un fait souhaitable qu'on doive garder lors de la traduction, car elle crée un fait d'insistance :

\begin{tabular}{|c|c|}
\hline M. Mandour & فأسرعي ، أسرعي عنهم \\
\hline El-Waghiche & فتدفقي، تدفقي لهم \\
\hline
\end{tabular}

Le traducteur doit essayer, autant que possible, de saisir les idées principales et de les reformuler par les termes convenables et adéquats dans la langue d'arrivée mais sans rien perdre, ni l'humour, ni le style du texte original. Les deux traducteurs ont équitablement saisi le sens de départ, mais avec certaines différences sémantiques. Mandour a traduit le verbe «couler» par un mot voisin qui donne le même sens, surtout, dans ce », tandis que El-Waghiche a trouvé le mot أسرع contexte « ». تدفقت

La répétition peut aussi véhiculer et mettre en valeur une idée

Ex : Aimons donc, aimons donc! (v. 33)

Avec l'effet d'écho, la voix de l'amante est prolongée et dédoublée : "aimons donc, aimons donc ». L'impératif «aimons donc ! » est repris deux fois sans modification. La répétition crée un rythme, et renforce l'idée, la sensation, l'émotion. Ces deux structures binaires insistent sur la nécessité impérieuse de profiter du temps présent. Les traducteurs ont manifestement tenu en compte des répétitions de la langue française.

\begin{tabular}{|l|l|}
\hline Mandour & فلنحب إذن فلنحب:
\end{tabular}

On rend la répétition telle quelle en arabe, lorsque l'effet stylistique lui parait digne d'être conservé. La répétition donne au style un caractère spontané, authentique. C'est une façon de nuancer la pensée. Elle contribue également à donner au vers plus de force, plus d'émotion. Un terme judicieusement répété 
fixe l'attention sur l'idée. Les traducteurs trouvent le sens approprié, mais chacun à sa manière. Voici la version de ElWaghiche :

El-Waghiche

A l'inverse de Mandour, El-Waghiche a recours à la synonymie pour nuancer son style de traduction. Gardant la répétition, il rend le verbe " aimons », repris deux fois, tantôt par

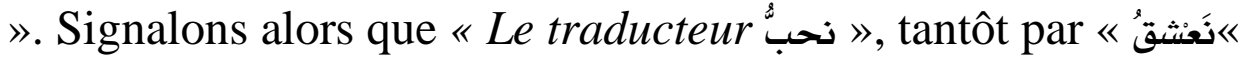
doit savoir aussi actualiser l'atmosphère du poème, sinon son sujet, sans porter atteinte sans doute à son contenu sémantique, à sa valeur phonique et à sa dimension universelle. Le traducteur peut ajouter une certaine couleur au poème qu'il traduit mais sans faire abstraction du caractère propre à la création poétique s" $»$.

La répétition peut être abusive, ou avoir une valeur rhétorique. Si elle est abusive elle devient une faute de langue et nous donne un style malhabile et un vocabulaire indigent.

\subsection{Le parallélisme}

Le parallélisme est un procédé de construction consistant à «la répétition d'un segment phrastique semblablement construit et d'une longueur similaire ${ }^{\xi \uparrow}$ ». Il est fondé principalement sur la coordination et sur la juxtaposition de deux phrases, de deux syntagmes, ou de deux vers pareillement construits. Contrairement au chiasme, il correspond au schéma A B / A B. Prenons cet exemple :

L'homme n'a point de port, le temps n'a point de rive.
(v.35)
$\mathrm{A}$
$\mathrm{B}$
A

B

¿) KAYRA, E. (1998). Le langage, la poésie et la traduction poétique ou une approche scientifique de la traduction poétique. Meta, 43(2), 254-261.

https://apropos.erudit.org/fr/usagers/politiquedutilisation/

«q ) https://fr.wikipedia.org/wiki/Parallélisme_(rhotorique) , consulté le 15/03/2018 
Dans ce vers, les deux noms communs (A) «l'homme - le temps »se répondent, de même que les deux groupes verbaux (B) " n'a point de port - n'a point de rive ». Alors, le parallélisme est une succession de deux groupes de mots de même construction.

En fait, le port n'existe pas, les rives non plus. On dirait que le fleuve du temps a tout englouti. Cependant, on ne peut jamais contrôler le temps. Le poète doit vivre dans un monde dont "l'homme n'a point de port, le temps n'a point de rive ». Car le temps s'échappe sans arrêter sa course. En tant que cette figure n'existe pas en arabe, les traducteurs ont recours à la rendre, tantôt littéralement, tantôt librement en imitant la même construction du vers original :

\begin{tabular}{|c|c|}
\hline M. Mandour & فالإنسان لا مرفأ له ، والزمن مـاله من شاطئ \\
\hline El-Waghiche & 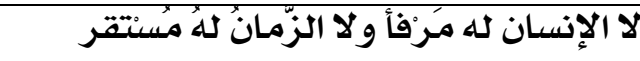 \\
\hline
\end{tabular}

Le parallélisme peut être renforcé par l'antithèse et l'anaphore quand la répétition de la construction se prolonge de la même manière.

Qu'il soit dans ton repos, qu'il soit dans tes

Beau lac, et orages,(v.53)

dans l'aspect de tes riants coteaux, (v.54)

Et dans ces noirs sapins, et dans ces rocs

$$
\text { sauvages }(v .55)
$$

Qui pendent sur tes eaux! (v.56)

Voyons à présent ce que nous dirions dans notre propre langue dans une situation analogue dans laquelle le parallélisme n’apparaît pas :

\begin{tabular}{|c|c|}
\hline M. Mandour & 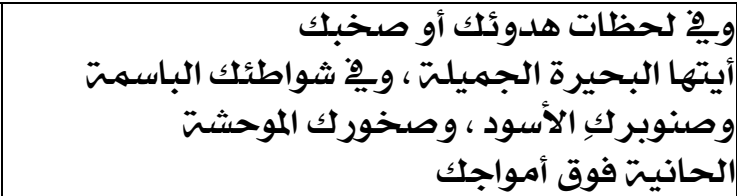 \\
\hline El-Waghiche & 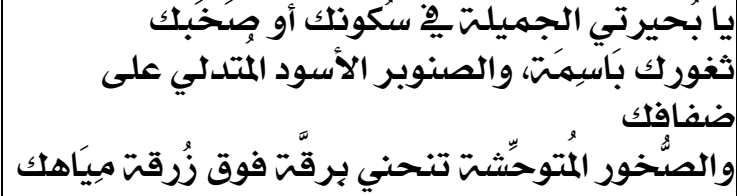 \\
\hline
\end{tabular}


Il peut également être renforcé par des phrases

exclamatives qui traduisent à la fois l'étonnement du poète et ses sentiments, de construction tels que :

"Quoi! Passés pour jamais! Quoi! Tout entiers perdus! $\gg(v .42)$

L'exclamation est rendue en arabe, parfois par son équivalent, parfois par l'interrogation :

\begin{tabular}{|c|c|}
\hline M. Mandour & أهكذا تمر إلى الأبد ؟ أهكذا يضيح كل شيء \\
\hline El-Waghiche & 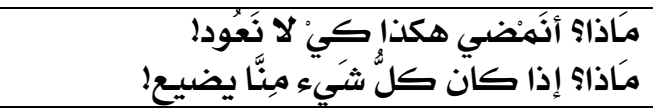 \\
\hline
\end{tabular}

\subsection{L'anaphore}

L'anaphore est « un procédé rhétorique qui consiste à reprendre un même mot ou groupe de mot au début d'énoncé successif afin de mettre en relief l'expression ainsi répétée" ». Alors, elle consiste à répéter un mot ou une expression au début d'une phrase ou d'un vers. Il doit être de la même catégorie grammaticale. Cette figure sert à insister sur une idée et la mettre en relief. Alors, quand on dit la même catégorie grammaticale, c'est-à-dire on ne peut pas faire l'anaphore avec un verbe plus un adjectif plus un nom. On doit voir soit la suit avec un nom ou bien avec un adjectif, ou bien avec un verbe. On ne peut pas mêler, par exemple, trois natures grammaticales. Et quand on parle de la mise en relief, c'est pour bien sûr valoriser une idée quand on utilise cette anaphore.

Les anaphores confèrent en outre un certain rythme au vers, ce qui engendre un effet musical. Elles sont plus courantes en français qu'en arabe, ce qui pose au traducteur un problème

•. ) DELISLE, J., La traduction raisonnée, Op. cit. p. 459 
constant. Doit-il éviter ces répétitions en arabe ou bien les conserver pour rendre un effet voulu par le poète ? Là, c'est l'intention du poète qui doit être respectée. Il faut essayer de déterminer si les anaphores sont voulues ; si elles pourraient contribuer à un effet stylistique. Si c'est le cas, il est préférable de les conserver en arabe. Jetons alors un coup d'œil sur ces exemples :

Gardez de cette nuit, gardez, belle nature, (v.51) Au moins le souvenir! (v.52)

Le poète s'adresse à la nature pour lui demander d'immortaliser ses souvenirs qu'il a vécus auprès d'elle avec son amante. Pour cela, il a recours à l'utilisation de l'anaphore du verbe impératif « gardez » repris deux fois pour créer la force de l'expression. Inlassablement, le poète essaye d'attirer la compassion et la piété de son lecteur vis-à-vis de cette situation tragique. Une anaphore dans le texte de départ ne se traduit pas nécessairement par une anaphore dans la langue du retour. Les fonctions de l'anaphore ne sont pas toutes les mêmes en français et en arabe. La comparaison du texte français avec sa traduction arabe nous fait constater que les traducteurs ne se sentent pas toujours tenus de rendre en arabe les anaphores, pourtant très fréquentes en français, comme dans l'exemple suivant :

\begin{tabular}{|c|c|}
\hline M. Mandour & الجميلتِي من هذه الأليلتّ، أيتها الطبيعت \\
\hline El-Waghiche & 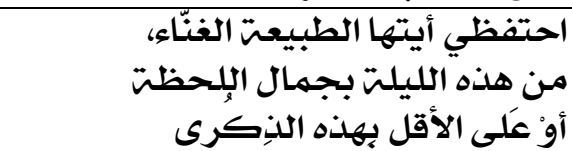 \\
\hline
\end{tabular}

Les traducteurs ont évité les répétitions. Ils ont eu recours à l'ellipse.

Les anaphores fréquentes tout au long du poème confèrent au poème un style évocateur. Les souhaits du poète sont répétés 
comme une évocation "Qu'il soit », "qu'il soit », "Qu'il soit », "Que le vent», "Que les parfums », "Que tout ce qu'on entend ». C'est ce qu'on appelle anaphore rhétorique, c'est-à-dire répétition d'un même mot en tête de vers. Cette répétition augmente une intensité jusqu'à la pointe finale.

Qu'il soit dans ton repos, qu'il soit dans tes orages, (v.53) Qu'il soit dans le zéphyr qui frémit et qui passe, (v.57)

Là, voyons très bien que la construction "Qu'il soit » se répète trois fois, et c'est toujours au début de la proposition. Ce qui crée bien sûr une anaphore pour montrer que le souvenir est de partout dans la nature. Les traducteurs rendent assez librement les anaphores si chères à la poésie française. Généralement, ils ne les transposent telles quelles en arabe que lorsque l'effet stylistique leur parait digne d'être conservé.

\begin{tabular}{|c|c|}
\hline \multirow[t]{2}{*}{ M. Mandour } & فِّْ لحظات هدوئلك أو صخبك \\
\hline & \\
\hline & كي \\
\hline
\end{tabular}

Les deux traducteurs ne rendent pas l'anaphore française » reprise deux 2 en arabe. Ils l'ont rendue par la préposition « fois en arabe et qui joue le rôle de l'anaphore en français. C'est pour valoriser une idée, créer une certaine musicalité et un certain rythme.

De plus, la persistance du poète est marquée par l'accumulation des propositions exposant ses vœux introduits par «Que» :

Que le vent qui gémit, le roseau qui soupire, (v.

Que les parfums légers de ton air embaumé, (v. 
(v. 63)

Que tout ce qu'on entend, l'on voit ou l'on respire,

Et là, il y a une anaphore avec "Que»: (Que le vent), (Que les parfums), (Que tout ce qu'on entend). Nous voyons qu'il existe une insistance sur la force de l'idée, et il existe le rythme aussi qui est très important. L'anaphore crée un effet musical, et donne une dynamique aux vers. Mais, elle est absente dans la traduction arabe :

\begin{tabular}{|c|c|}
\hline M. Mandour & 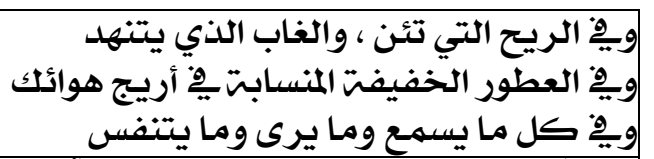 \\
\hline El-Waghiche & 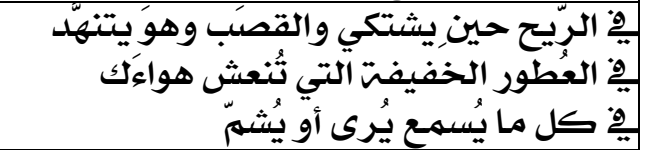 \\
\hline
\end{tabular}

Les traducteurs rendent l'anaphore en arabe par la » répétée trois fois. Cependant, ce procédé ne थ̈préposition « porte pas tort à la compréhension du message du texte cible. L'arabe n'épreuve pas la même répugnance que le français à l'égard de la répétition. Le français tolère mieux que l'arabe la répétition lexicale. La fonction de l'anaphore n'est pas la même dans les deux langues en présence.

\subsection{L'accumulation}

C'est une succession de plusieurs termes dans la visée d'insister sur une idée.

\section{Ô lac! Rochers muets! Grottes! Forêt}

obscure! (v. 49)

Là, il y a une accumulation. Le poète s'adresse à tous les éléments de la nature : "Rochers muets Grottes! Forêt obscure !» pour frapper l'imagination, pour influencer le lecteur et pour montrer les qualités de ce lac. 
Dans ce vers, le poète a recours à l'accumulation reprenant le ton oratoire, et fait appel à nouveau au lac directement. Mais, là, l'apostrophe «Ô lac!» est suivie d'un appel aux «rochers », aux « grottes » et à la «forêt ». Le poète enferme dans le lac tous les éléments de l'environnement pour rendre son appel plus solennel. C'est une accumulation parfaite et très réussie parce qu'il va voir un effet certain sur le lecteur. Le fait de respecter des accumulations dans la langue de retour rend la traduction pertinente et appropriée.

\begin{tabular}{|c|c|}
\hline M. Mandour & لحالكتا البحيرة ، أيتها الصخور الصامتتة، والكهوف والغابت \\
\hline El-V & 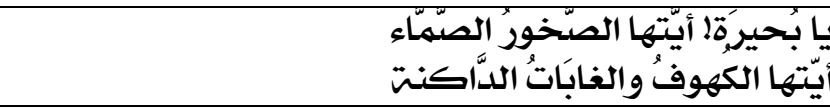 \\
\hline
\end{tabular}

On utilise les figures de style pour attirer l'attention du lecteur, pour le séduire, pour le convaincre, pour frapper son imagination et pour lui transmettre une certaine vision du monde. C'est dans cet optique là qu'on utilise les figures de style. Elles représentent aussi un cas par rapport à la langue, fonctionnel. Elles visent l'expressivité. C'est l'aspect poétique du style. Ce qui engendre une symbiose parfaite entre le fond et la forme".

\section{Les traits de la versification}

Pour la régularité de versification, le poème est formé de seize quatrains composés soit de trois alexandrins ${ }^{\text {or }}$ et un hexasyllabe ${ }^{o r}$, soit d'alternance alexandrin-hexasyllabe. Les vers sont plus courts et plus chargés d'émotion. Ils sont marqués par un rythme harmonique, et musical approprié à l'élégie lamartinienne. La question la plus ardue lors de la traduction

") https://nanopdf.com/download/les-figures-de-styles-et-leurs-effets consulté le $15 / 12 / 2017$

or) En poésie française, un alexandrin est un vers de douze syllabes ; https://fr.vikidia.org/wiki/Alexandrin

$\left.{ }^{\circ}\right)$ En poésie française, un hexasyllabe est un vers de six syllabes 
poétique est celle de la versification "rime, rythme, allitération et assonance ». Ricœur considère comme la difficulté majeure dans la traduction de la poésie : "l'union inséparable du sens et de la sonorite ${ }^{o f} »$ qui caractérise la poésie. De là, la comparaison sur le plan de la versification s'avère impossible. Alors, on pourrait admettre que «la traduction totale n'est pas possible en raison de l'immanence des codes d'une langue, irréductibles les uns des autres ${ }^{\circ \circ} »$. Mais, il est habituellement possible de rendre la signification d'un texte poétique indépendamment de ses traits formels et harmoniques. Pour bien garder le sens, on sacrifie la rime, le rythme ou la transposition de la même forme métrique original.

Pour nous, le respect de la disposition en strophe est indispensable. Elle crée un effet visuel « le vers n'est pas la donnée première de notre réception de ces textes, car pour les yeux comme pour l'oreille, cet élément de base est la strophe ${ }^{\circ »}$.

\subsection{La rime}

En général, on trouve la rime en fin de vers, ou, quelquefois, à la césure. La rime est croisée « $\mathrm{ABAB} »$ avec alternance d'une rime féminine "terminée par un " $e$ " mиеt, elle est plus douce », et d'une rime masculine. Le poète a recours à la rime croisée pour renforcer l'opposition entre le malheur et le bonheur ; et donner une image merveilleuse. Garder la rime est une question impraticable surtout lorsqu'on parle de deux langues tout à fait différentes, et aucune convergence parentale n'existe entre les deux. "Trop souvent la traduction rimée tourne alors au pastiche et son infériorité par rapport à l'original

${ }^{\circ)}$ RICOEUR, P, Sur la traduction, Op. cit., p.12

○) LAROSE, Robert, (1978), L'analyse structurale en traduction poétique. Meta, 23(1),

47-62. doi :10.7202/003411ar, consulté le 12 mars 2018 05:55

$\left.{ }^{\circ}\right)$ MESCHONNIC, H., Poétique du traduire, Op. cit., p. 265. 
devient trop éclatante ${ }^{o v} »$. Rendant d'une langue à l'autre, on pourrait s'éloigner du sens original pour faire revivre la musicalité. Mais, il est possible de rimer sans forcer le sens. Dans sa version rimée, El-Samahy a bien transféré le sens. Il a équitablement respecté les caractères du poème original. Il a rendu ce poème avec une perfection incroyable. Sa traduction arabe, fidèle et très bien rimée, est aussi belle que l'original.

Un bon exemple d'une traduction rimée faite par ElSamahy dans ces vers :

\begin{tabular}{|c|c|}
\hline Lamartine & $\begin{array}{l}\text { "Aimons donc, aimons donc ! de l'heure fugitive, } \\
\text { Hâtons-nous, jouissons! } \\
\text { L'homme n'a point de port, le temps n'a point de } \\
\text { rive; } \\
\text { ll coule, et nous passons!" }\end{array}$ \\
\hline El-Samahy & 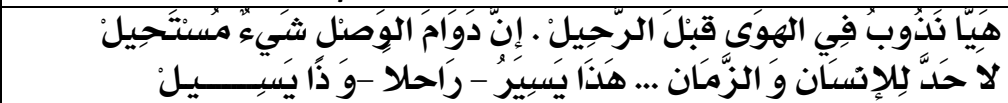 \\
\hline
\end{tabular}

Là, El-Samahy est un traducteur doué et expérimenté et nous donne une traduction à la fois fidèle, rimée, brillante et agréable à lire. Lisant sa traduction, on est stupéfait et reste confondu d'admiration devant sa perfection. Il n'omet rien, n'ajoute rien et ne déforme rien. Avec son goût très particulier et son vaste talent poétique, il rend parfaitement l'atmosphère du poème original, son rythme et sa musique. Tandis que Fayad, dans sa version, nous a donné une belle traduction rimée, mais il a déformé le sens en raison de conserver la rime et faire revivre la musique.

\begin{tabular}{|l|l|}
\hline Lamartine & $\begin{array}{l}\text { Ainsi, toujours poussés vers de nouveaux } \\
\text { rivages, } \\
\text { Dans la nuit éternelle emportés sans }\end{array}$ \\
\hline
\end{tabular}

^v ) ELLRODT, Robert, «Comment traduire la poésie ?», Op. cit., consulté le 28 janvier 2018. URL : http://palimpsestes.revues.org/247 


\begin{tabular}{|c|c|}
\hline & $\begin{array}{l}\text { retour, } \\
\text { Ne pourrons-nous jamais sur l'océan des } \\
\text { ages } \\
\text { Jeter l'ancre un seul jour? }\end{array}$ \\
\hline N. Fayad & 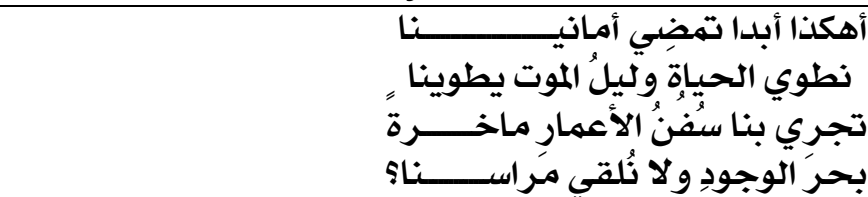 \\
\hline
\end{tabular}

Le traducteur a admirablement joué de la musique. Sa traduction possède la même douceur pathétique et sentimentale que l'original. Il respecte la strophe et les caractères des vers sources. Formellement, c'est une belle traduction, et qui est plus rimée. Mais est-elle fidèle ? Cette traduction me parait inadéquate et décevante. Retranchant et rajoutant des termes inexistant dans le poème original, le traducteur s'écarte, un peu, du sens des vers sources parce qu'il cherche toujours la rime. A ) est là notre avis, la rime est responsable de la catastrophe : ( ). Fayad recrée le poème, et en fait مراسيـــpour rimer avec ( quelque chose de très beau, mais de très différent du texte source. En fait, les traductions rimées " séduisent le regard, mais elles sont, pour l'affamé, fades comme des potirons ${ }^{\text {}} »$.

Par contre, il est souvent intéressant de traduire en prose sans déformer et perturber le sens, telle la version de Mandour et celle d'El-Waghiche. Leur traduction n'est pas rimée, mais elle est à la fois belle et remarquablement exacte. Nous voyons que le bon traducteur doit " connaître les dessous des choses, lire entre les lignes, accorder la priorité au sens, faire fi de la littéralité,

^) MAISONNEUVE, R. (1978). La musique du mot et du concept, ou certains problèmes de traduction poétique, Meta, 23(1), 73-85. doi :10.7202/003542ar, consulté le 21 mars $201805: 56$ 
rechercher des équivalences tant dynamiques que

contextuelles ${ }^{\circ{ }}$. Voyons leur traduction :

\begin{tabular}{|c|c|}
\hline Lamartine & $\begin{array}{l}\text { Un soir, t'en souvient-il ? Nous voguions en } \\
\text { silence; } \\
\text { On n'entendait au loin, sur l'onde et sous les } \\
\text { cieux, } \\
\text { Que le bruit des rameurs qui frappaient en } \\
\text { cadence } \\
\text { Tes flots harmonieux. }\end{array}$ \\
\hline $\begin{array}{l}\text { M. } \\
\text { Mandour }\end{array}$ & 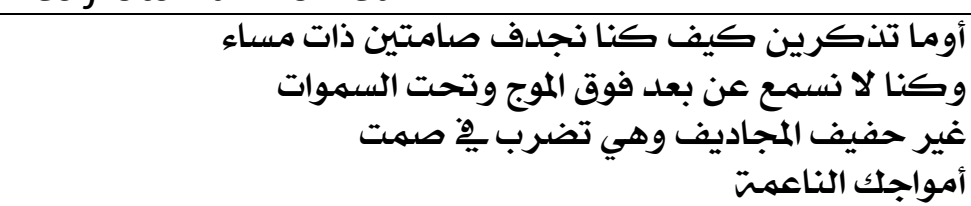 \\
\hline El-Waghiche & 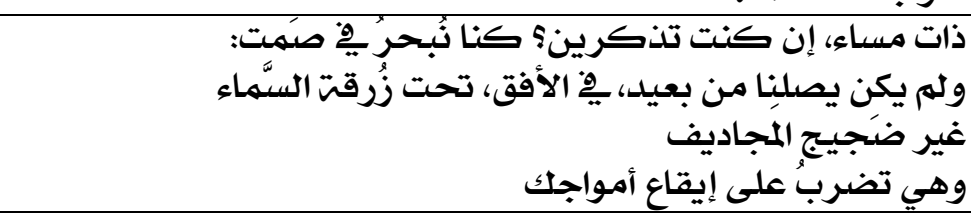 \\
\hline
\end{tabular}

Bien gardant le sens, Mandour et El-Waghiche sacrifient un élément essentiel du poème. Ils sacrifient le rythme et la musique du vers. Leur traduction en prose ne contient pas de grandes satisfactions esthétiques et artistiques et ne donne pas l'impression d'un poème. Ils accordent la priorité au sens et font fi de la rime.

Traduisant la poésie, on a alors deux solutions : on garde la rime, et c'est un fait très périlleux, ou on ne cherche pas la rime, c'est-à-dire «rimer ou ne pas rimer». On rime, on garde la musique du vers, mais on peut être infidèle. On ne rime pas, on perd la musique du vers, mais on peut être fidèle. A mon avis, le traducteur doué et expérimenté doit apprendre : "à se connaître lui-même et à mobiliser ses forces et ses possibilités et à les 
accroître systématiquement par l'exercice [...], à affronter les difficultés, à vaincre les obstacles [...], à reconnaître la nature des poètes, à se rendre compte de leurs points communs, de leurs divergences [...] n à reconnaître la nature du langage poétique, avec ses différents aspects, ses variations, son évolution [...], à reconnaître le génie de deux langues, la différence de leur organisation, de leur orientation, de leurs ressources, de leur évolution" ".

\subsection{Allitération et Assonance}

Ayant une fonction esthétique et flattant l'oreille, ces deux éléments « sonores » jouent souvent un rôle de construction du vers et participent de la musique du poème, mais aussi, ils se mettent «en relation avec des effets sémantiques" ». Le poème $\mathrm{du}$ «lac » joue beaucoup sur les sonorités : tout au long du poème, il existe nombreuses allitérations et assonances.

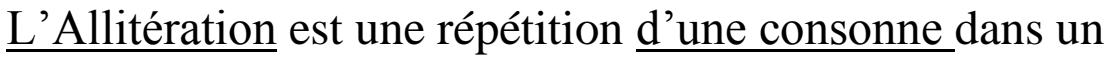
but expressif. Son rôle essentiel est d'engendrer une harmonie imitative, de donner un rythme au vers et de créer une trame sonore du poème.

\begin{tabular}{|l|l|}
\hline Lamartine & $\begin{array}{l}\text { "Ainsi, toujours poussés vers de nouveaux } \\
\text { rivages » }\end{array}$ \\
\hline M. Mandour & \multicolumn{1}{|c|}{ أنظل هكذا منساقين أبدا إلى شواطئ جديدة؛ }
\end{tabular}

L'allitération est clairement construite par la répétition de la consonne [v] et de la consonne [s] : ce qui convoque le pouvoir des forces de la destinée qui portent les être humains comme une vague irrésistible. Signalons que la plupart des allitérations, tout au long du poème, sont en [R].

7) SCHNEIDER, A. (1978). La traduction poétique. Meta, 23(1), 20-36. doi:10.7202/004051ar

") GARDES-TAMINE, J., MOLINO, Jean, Introduction à l'analyse de la poésie, Op. cit. p. 84 . 
Les enjeux de la traduction poétique Le cas du « Lac » de Lamartine

\begin{tabular}{|l|l|}
\hline Lamartine & $\begin{array}{l}\text { "Le flot fut attentif } \\
\text { " فأنصت الموجج }\end{array}$ \\
\hline M. Mandour &
\end{tabular}

L'allitération de la consonne [f] évoque la fluidité de l'élément aquatique.

L'Assonance est une répétition d'une voyelle dans un but expressif. Son rôle principal est de créer des échos entre les mots. Combinée à l'allitération, elle crée une musique des vers et met en évidence une unité de sons".

\begin{tabular}{|c|c|}
\hline Lamartine & $\begin{array}{l}\text { "Laissez-nous savourer les rapides } \\
\text { délices" }\end{array}$ \\
\hline El-Waghiche & خَلينا نستمتع بَأفراحنا أجمل لحظات أيّامنا \\
\hline
\end{tabular}

L'allitération du son consonne [s] et assonance du son voyelle [ou].

L'assonance du son voyelle [ou] conjuguée à l'allitération des consonnes [v] et [s] crée une musique évocatrice du mouvement chronologique qui roule l'existence humaine dans ses rouages.

Si la sonorité se révèle plus organisée le poème source, on ne retrouve pas de véritable progression de sonorité en arabe. Les deux langues en question n'ont pas recours aux mêmes sons, du fait des différences entre les langues. Ces figures de style (allitération et assonance) qui mettent en jeu des sonorités identiques, sont absentes en arabe. Sans porter atteint à la transmission de l'idée du poème original, le traducteur ne maintient pas l'allitération et l'assonance. Rarement qu'un effet sonore puisse être rendu spontanément d'une langue à l'autre.

\subsection{Le rythme}

"r ) www.espacefrancais.com/la-versification/ consulté le 05/01/2018 
Le rythme scande le poème en faisant sentir le nombre de syllabe et des pièds.

\subsubsection{L'enjambement}

La phrase dépasse sur le vers suivant. "La phrase dépasse la limite du vers et continue sur le vers suivant.

[..] L'enjambement est souvent utilisé pour mettre en relief un mot ou pour créer un effet de surprise $e^{\text {tr }}$ ». dans le poème en question, les enjambements sont nombreux notamment en fin de strophe (v. 7-8, 11-12, 15-16, 17-18, 23-24, 31-32, 39-40, 47-48, 55- 56, 59-60) pour rallonger les vers.

silence ;(v.13)

Un soir, t'en souvient-il ? Nous voguions en

On n'entendait au loin, sur l'onde et sous les cieux, (v.14)

Que le bruit des rameurs qui frappaient en cadence (v.15)

Tes flots harmonieux. (v.16)

Dans ce quatrain, la dernière phrase se prolonge jusqu'au dernier vers. C'est ce qu'on appelle enjambement. La phrase dépasse la limite du vers et continue sur le vers suivant. Il se rapporte à deux vers consécutifs qui doivent être lus sans discontinuité, comme s'il s'agissait d'un seul vers ${ }^{\text {\} . ~ L e ~ f a i t ~ d e ~ }$ respecter l'enjambement dans le texte cible nous donne incontestablement une traduction significative et fidèle :

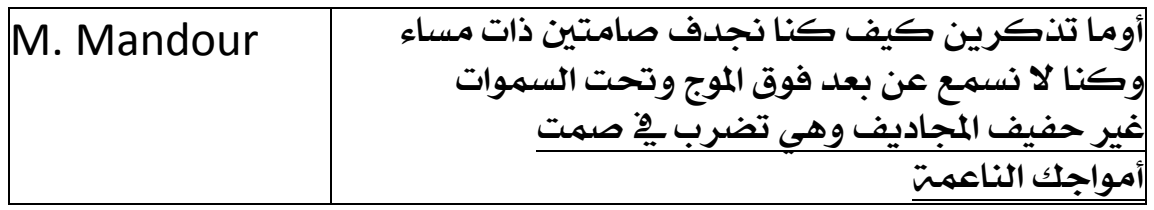

"r) https://www.etudes-litteraires.com/figures-de-style/enjambement.php consulté le $11 / 01 / 2018$

") www.bacdefrancais.net/rime.php consulté le 15/01/2018 
Les enjeux de la traduction poétique Le cas du « Lac » de Lamartine

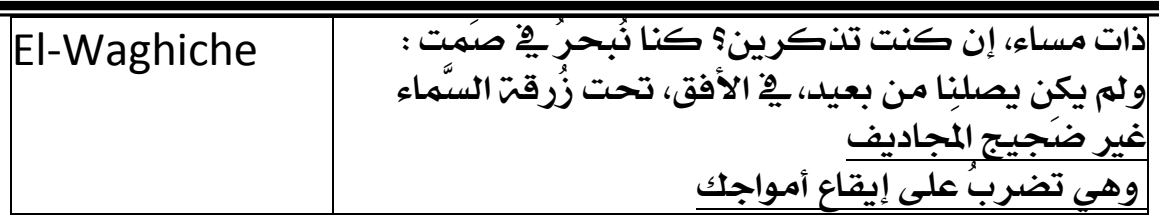

On remarque, dans les deux versions, que la phrase ne s'arrête pas à la fin du vers, mais déborde jusqu'à la fin du vers suivant. L'enjambement crée un équilibre rythmique dans les deux vers. En effet le débordement syntaxique d'un vers sur le vers suivant «atténue la pause en fin de vers, ou même l'abolit presque totalement, créant un effet d'allongement ou de déstructuration ${ }^{\text {"0 }}$.

\subsubsection{Rejet et contre rejet}

Lorsque le sens du vers se termine au début du vers suivant, il existe un rejet

On n'entendait au loin, sur l'onde et sous les cieux, Que le bruit des rameurs qui frappaient en cadence Tes flots harmonieux

Le rejet est constitué par un élément bref (quelques syllabes suivies d'une coupe marquée) lié syntaxiquement au vers précédent mais reporté ( « rejeté ») au début du vers suivant pour « créer un effet de soulignement et de surprise après l'atténuation forte de la pause traditionnelle en fin de vers "». Le fait de respecter le rejet dans la langue cible rend la traduction pertinente et appropriée. Et produit un effet rythmique

\begin{tabular}{|c|c|}
\hline M. Mandour & 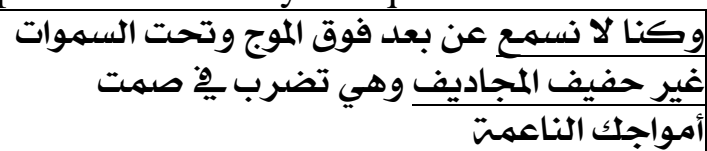 \\
\hline El-Waghiche & 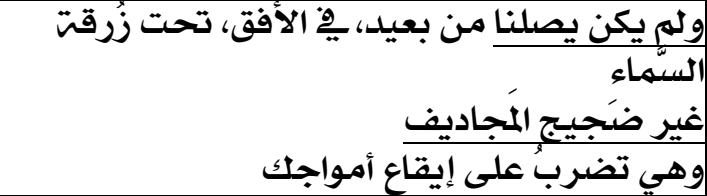 \\
\hline
\end{tabular}

70 ) MAZALEYRAT, Jean, Eléments de métrique française, A. Colin, Paris, 1974, p.122

") Ibid., p.127 
》 lié غير حفيف - غير ضَجيجCet exemple montre qu'un élément « syntaxiquement et sémantiquement au vers précédent est placé au début du deuxième vers.

Tandis qu'il y a contre-rejet quand la phrase commence à la fin du vers précédent :

Je dis à cette nuit : Sois plus lente; et l'aurore Va dissiper la nuit.

Le contre-rejet est constitué par un élément bref (quelques syllabes précédées d'une coupe marquée) lié syntaxiquement et sémantiquement au vers suivant "mais mis en relief en jouant sur l'atténuation forte de la pause

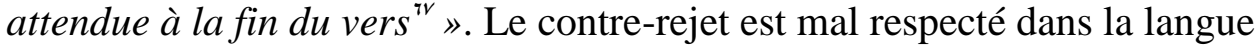
d'arrivée.

\begin{tabular}{|c|c|}
\hline M. Mandour & والفجرل لهذا الليل تهـل الليل \\
\hline El-Waghiche & 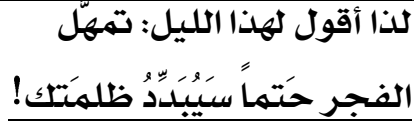 \\
\hline
\end{tabular}




\section{Conclusion}

Au terme de cette étude, on peut affirmer que la poésie est intrinsèquement difficile à traduire, mais non impossible. Cette difficulté provient de la nature même de la poésie, vu qu'elle a des caractéristiques spécifiques qui compliquent la traduction. La langue poétique est une langue musicale car le poète joue admirablement de la musique. Elle se reconnaît également par ses images. En détournant les mots de leur sens habituel ou en les associant de façon inattendue, elle nous pose des problèmes de traduction. On a envisagé de mettre en regard de différentes traductions du poème étudié afin de comparer la manière dont il a été rendu par les traducteurs. De là, la présente recherche aborde les enjeux liés à la traduction poétique.

Le lac de Lamartine est fondé sur une série de métaphores, de périphrases, de personnifications, de répétitions, d'antithèses, d'accumulations et d'anaphores pour souligner l'insistance du poète sur le fait que le lac conserve son souvenir et exprime son angoisse devant la fuite du temps, et reflète son désir d'immortaliser ses souvenirs et son amour.

Concernant la transposition des figures de style dans la langue du retour, doit-il les rendre littéralement ou librement ? Dans toutes les pages précédentes, nous avons déjà répondu. $\mathrm{La}$ question ne s'établit pas entre une traduction littérale ou une traduction libre, mais entre une traduction exacte et une traduction inexacte. En fait, le traducteur doit rendre dans une autre langue le contenu et les connotations des figures de style. Il doit en même temps garder autant que possible ses traits formels. Parfois, les figures de style, d'une langue à l'autre se correspondent absolument ou à peu près. Si les deux langues en présence ont des traditions communes, alors, le transfère d'une image par son équivalent ne pose aucune difficulté. On peut également rester littéral tant qu'on ne fait pas violence à la langue du retour. Mais, si ce n'est pas possible de rendre les 
figures littéralement, on n'hésite pas à transférer le sens, en d'autres termes, à recourir à une équivalence. Au cas où, on doit essayer de trouver une équivalence et, si n'est pas possible, on s'attache à traduire l'idée.

La question de la traduction du poème en prose nous a tout particulièrement intéressé. Il est parfaitement admissible de traduire un poème en prose libre. Il faut en traduisant respecter la valeur signifiante du texte source. Certes, il y a une traduction en vers rimés, mais, le traducteur est conduit à sacrifier parfois l'exactitude au profit de la fluidité.

La traduction de Fayad est formellement la meilleure. Mais, il s'écarte, en grande partie, du sens des vers sources parce qu'il cherche toujours la rime. La garde de la rime ne peut se faire que par une tricherie perpétuelle qui amène le traducteur à s'éloigner beaucoup du texte original. Par contre, la traduction d'El-Samahy est en général fidèle, agréable à lire et brillante. Gardant la rime, on est stupéfait et reste confondu d'admiration devant sa perfection. Avec son goût très particulier et son vaste talent poétique, il rend parfaitement l'atmosphère du poème original, son rythme et sa musique. Bien gardant le sens, Mandour et ElWaghiche sacrifient un élément essentiel du poème. Ils sacrifient le rythme et la musique du vers. Leur traduction en prose ne contient pas de grandes satisfactions esthétiques et artistiques et ne donne pas l'impression d'un poème. Ils accordent la priorité au sens et font fi de la rime.

Vu la difficulté que pose fréquemment la traduction poétique, le traducteur est tenu d'avoir recours aux solutions possibles pour rendre un poème rimé : "Rimer ou ne pas rimer ». On rime, alors on garde la musique du vers, mais on peut être infidèle. On ne rime pas, on perd la musique du vers, mais on peut être fidèle. Il faut équitablement traduire la poésie de façon à conserver son caractère esthétique et son aspect pathétique. Mais, le traducteur d'un poème est conduit à choisir entre l'exactitude et la recherche d'un effet esthétique. 


\section{Bibliographie \\ Corpus}

E, les Méditations Poétiques, 1. LAMARTIN

www.poetes.com/textes/lam_nmed

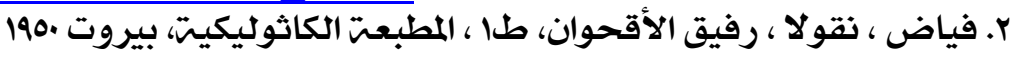

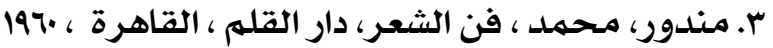

. Idris El-Waghiche \&

http://www.ahewar.org/debat/show.art.asp?aid=502566

. Mohamed El-Samahy •

http://www.matarmatar.net/threads/4931/

\section{Ouvrages francais}

1. AURY, Dominique, «Préface », Les problèmes théoriques

de la traduction, de George Mounin, Paris, Gallimard, 1963

2. BAUDELAIRE, Charles. Histoires grotesques et sérieuses.

Michel Lévy frères, Paris, 1865

3. BERMAN, Antoine, «La traduction et la lettre ou

L'auberge du lointain », Seuil, Paris, 1999.

4. BONHOMME, Marc, Les figures clés du discours, Seuil, Paris, 1998.

5. DUMARSAIS, César Chesneau, Des tropes, Flammarion, Paris, 1988.

6. FONTENIER, Pierre, Les figures du discours, Flammarion, Paris, 1977.

7. DELISLE, J., La traduction raisonnée, PUO, Canada, 2010.

8. GARDES-TAMINE, J., HUBERT. M. C., Dictionnaire de critique littéraire, A. Colin, Paris, 2002.

9. GARDES-TAMINE, J., MOLINO, Jean, Introduction à

l'analyse de la poésie, PUF, Paris, 1982 ;

10. GILE, D., La traduction, la comprendre, l'apprendre, PUF, Paris 2005, p. 56 
11. KUNDERA, Milan, Les testaments trahis, Gallimard, Paris, 1993.

12. MAZALEYRAT, Jean, Eléments de métrique française,

A. Colin, Paris, 1974

13. MESCHONNIC, Henri, Poétique du traduire, Verdier, Paris, 1999

14. ONDRUSKOVA, Dana, la nature dans les recueils les méditations poétiques et les destinées de Lamartine et Vigny, Thèse de baccalauréat, Université de Masaryk À Brno, 2011.

15. OSEKI-DEPRE, Inès, Traduction \& poésie, Maisonneuve \& Larose, Paris, 2004

16. PIERRE, Jean, Euvres II, texte établi et présenté par Jean Starobinski, Mercure de France 1987.

17. RICOEUR, P., La Métaphore vive, Seuil, Paris, 1975

18. RICOEUR, P, Sur la traduction, Bayard, Paris, 2004

19. SELESKOVITCH D., \& LEDERER M., Interpréter pour traduire. Didier, Paris, 2001;

20. VINAY, DARBELNET, Stylistique comparée du français et de l'anglais, Didier, Paris, 2009.

\section{Ouvrages arabes}

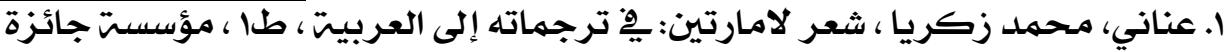

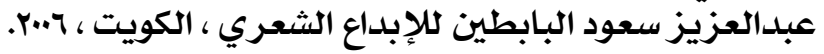

\section{Périodiques}

AMID, Abdallah, la fidélité en traduction double pour .' qu'elle soit belle et fidèle, Acte de Colloque, fac. Des sciences humaines, Al Azhar, le Caire, 2015. BATTAGLIA, Anna, GARDES-TAMINE, J., «Traduire •r la poésie : du mot au texte », in : Synergies Italie, 2010, $\mathrm{n}^{\circ} 6$.

ELLRODT, Robert, «Comment traduire la poésie? », .r Palimpsestes [En ligne], mis en ligne le 01 septembre 2008, consulté le 28 janvier 2018. URL : 
http://palimpsestes.revues.org/247; DOI :

10.4000/palimpsestes.247

BLANCHOT, M., Faux pas, Paris, Gallimard, 1943, cité . .

par G. Genette, La Littérature au second degré, in

Palimpsestes. Paris, Seuil, 1982

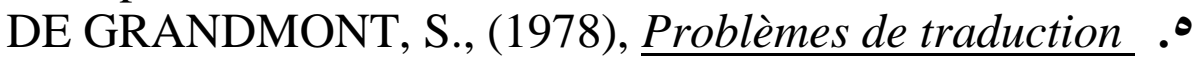

dans le domaine de la poésie chantée, Meta 23(1), 97-108.

doi : 10.7202/002959ar, consulté le 21 mars 201808 :56

MEHANNA, Ghadaa, Traduire sans trahir, colloque .7

AEPF, à Port Said « la traduction enjeux et intérêt, 21- 23

avril 2006.

KAYRA, E. (1998). Le langage, la poésie et la traduction .V poétique ou une approche scientifique de la traduction poétique. Meta, 43(2), 254-261.

https://apropos.erudit.org/fr/usagers/politiquedutilisation/

LAROSE, Robert, (1978), L'analyse structurale en.$^{\wedge}$

traduction poétique. Meta, 23(1), 47-62. doi :

10.7202/003411ar, consulté le 12 mars 2018 05:55

MAISONNEUVE, R. (1978). La musique du mot et du .9

concept, ou certains problèmes de traduction poétique,

Meta, 23(1), 73-85. doi : 10.7202/003542ar, consulté le 21

mars $201805: 56$

PENISSON, Pierre, «Le génie traducteur $»$, in : M. . .

Broda (éd.), La traduction-poésie, à Antoinen Berman,

Presses universitaires de Strasbourg, Strasbourg, 1999.

SCHNEIDER, A. (1978). La traduction poétique. . .'

Meta, 23(1), 20-36. doi : 10.7202/004051ar.

VAN HOOF, Henri, « recherche d'un modèle . $\quad$ ir

d'analyse en traduction », dans Méta, vol.16, 1971

\section{$\underline{\text { Dictionnaires }}$}

REY, A. et REY-DEBOVE J., Le Petit Robert, .'

dictionnaire de la langue française, Robert, Paris, 2001 


\section{Sitographgies}

http://www.bacdefrancais.net/le-lac-lamartine.php. .' consulté le 11/11/2017

, https://fr.wikipedia.org/wiki/Parallélisme_(rhotorique) .r consulté le 15/03/2018

https://apropos.erudit.org/fr/usagers/politiquedutilisation/ r consulté le 10/10/2017

consulté le 28 janvier http://palimpsestes.revues.org/247 . . 2018

consulté http://www.espacefrancais.com/la-versification/.$^{\circ}$ le $05 / 01 / 2018$

, https://www.comptoirlitteraire.com/docs/465-lamartine .7 consulté le 11/11/2017

https://nanopdf.com/download/les-figures-de-styles-et- .V leurs-effets, consulté le 15/12/2017

https://www.etudes-litteraires.com/figures-de- .A

style/enjambement.php, consulté le 11/01/2018

consulté le 15/01/2018www.bacdefrancais.net/rime.php .9 


\section{Annexe \\ Le lac de Lamartine et ses différentes traductions Traduction de Nicolas Fayad}

\begin{abstract}
Ainsi, toujours poussés vers de nouveaux rivages, Dans la nuit éternelle emportés sans retour, Ne pourrons-nous jamais sur l'océan des âges Jeter l'ancre un seul jour?

Ô lac ! l'année à peine a fini sa carrière, Et près des flots chéris qu'elle devait revoir, Regarde! je viens seul m'asseoir sur cette pierre Où tu la vis s'asseoir !

Tu mugissais ainsi sous ces roches profondes, Ainsi tu te brisais sur leurs flancs déchirés, Ainsi le vent jetait l'écume de tes ondes Sur ses pieds adorés.
\end{abstract}

Un soir, t'en souvient-il ? nous voguions en silence ; On n'entendait au loin, sur l'onde et sous les cieux, Que le bruit des rameurs qui frappaient en cadence Tes flots harmonieux.

Tout à coup des accents inconnus à la terre Du rivage charmé frappèrent les échos ; Le flot fut attentif, et la voix qui m'est chère Laissa tomber ces mots :

" Ô temps ! suspends ton vol, et vous, heures propices ! Suspendez votre cours : Laissez-nous savourer les rapides délices Des plus beaux de nos jours !

" Assez de malheureux ici-bas vous implorent, Coulez, coulez pour eux ;

Prenez avec leurs jours les soins qui les dévorent ; Oubliez les heureux.

" Mais je demande en vain quelques moments encore, Le temps m'échappe et fuit ;

Je dis à cette nuit : Sois plus lente; et l'aurore Va dissiper la nuit.

" Aimons donc, aimons donc ! de l'heure fugitive, Hâtons-nous, jouissons !

L'homme n'a point de port, le temps n'a point de rive ; Il coule, et nous passons ! "

Temps jaloux, se peut-il que ces moments d'ivresse,

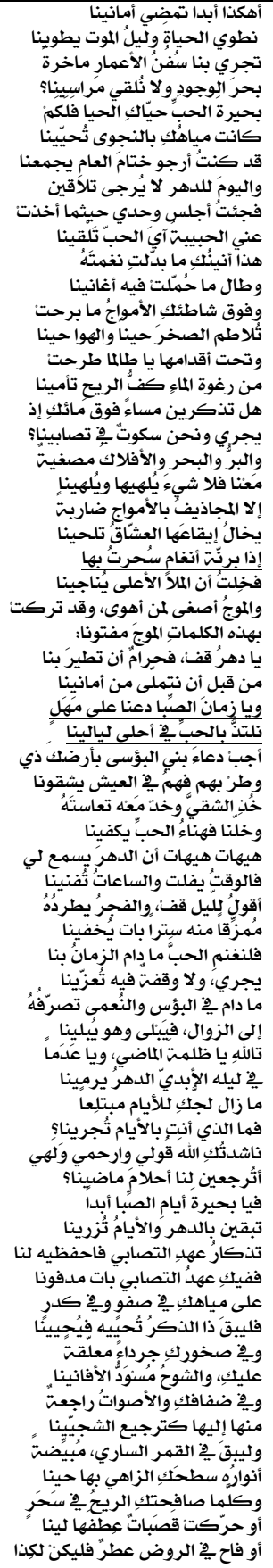


Où l'amour à longs flots nous verse le bonheur, S'envolent loin de nous de la même vitesse Que les jours de malheur?

Eh quoi ! n'en pourrons-nous fixer au moins la trace ? Quoi ! passés pour jamais ! quoi ! tout entiers perdus ! Ce temps qui les donna, ce temps qui les efface, Ne nous les rendra plus !

Éternité, néant, passé, sombres abîmes, Que faites-vous des jours que vous engloutissez ? Parlez : nous rendrez-vous ces extases sublimes Que vous nous ravissez?

Ô lac ! rochers muets ! grottes ! forêt obscure ! Vous, que le temps épargne ou qu'il peut rajeunir, Gardez de cette nuit, gardez, belle nature, Au moins le souvenir !

Qu'il soit dans ton repos, qu'il soit dans tes orages, Beau lac, et dans l'aspect de tes riants coteaux, Et dans ces noirs sapins, et dans ces rocs sauvages Qui pendent sur tes eaux.

Qu'il soit dans le zéphyr qui frémit et qui passe, Dans les bruits de tes bords par tes bords répétés, Dans l'astre au front d'argent qui blanchit ta surface De ses molles clartés.

Que le vent qui gémit, le roseau qui soupire, Que les parfums légers de ton air embaumé, Que tout ce qu'on entend, l'on voit ou l'on respire, Tout dise : lls ont aimé !

\section{La traduction de Mohamed Mandour}

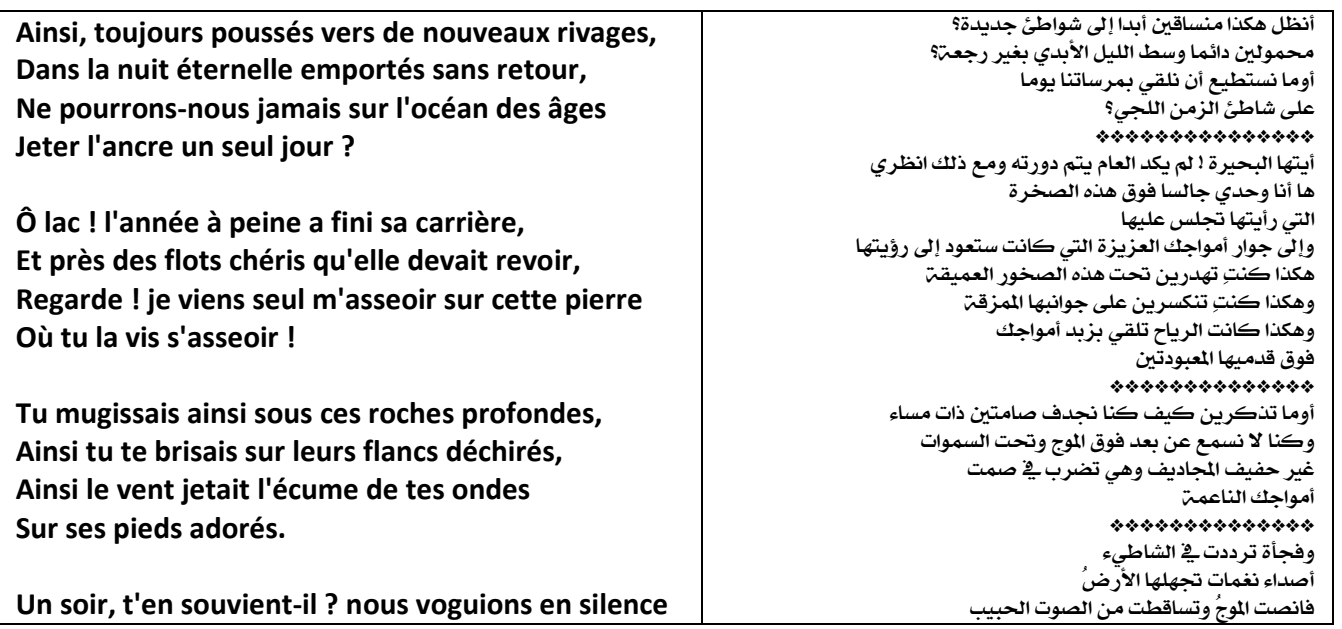


;

On n'entendait au loin, sur l'onde et sous les cieux, Que le bruit des rameurs qui frappaient en cadence Tes flots harmonieux.

Tout à coup des accents inconnus à la terre Du rivage charmé frappèrent les échos ; Le flot fut attentif, et la voix qui m'est chère Laissa tomber ces mots :

" Ô temps ! suspends ton vol, et vous, heures propices!

Suspendez votre cours :

Laissez-nous savourer les rapides délices

Des plus beaux de nos jours !

" Assez de malheureux ici-bas vous implorent, Coulez, coulez pour eux ;

Prenez avec leurs jours les soins qui les dévorent ; Oubliez les heureux.

" Mais je demande en vain quelques moments encore,

Le temps m'échappe et fuit ;

Je dis à cette nuit : Sois plus lente; et l'aurore Va dissiper la nuit.

" Aimons donc, aimons donc ! de l'heure fugitive, Hâtons-nous, jouissons !

L'homme n'a point de port, le temps n'a point de rive ;

Il coule, et nous passons ! "

Temps jaloux, se peut-il que ces moments d'ivresse,

Où l'amour à longs flots nous verse le bonheur, S'envolent loin de nous de la même vitesse Que les jours de malheur?

Eh quoi ! n'en pourrons-nous fixer au moins la trace?

Quoi ! passés pour jamais ! quoi ! tout entiers perdus!

Ce temps qui les donna, ce temps qui les efface, Ne nous les rendra plus !

Éternité, néant, passé, sombres abîmes, Que faites-vous des jours que vous engloutissez ? Parlez : nous rendrez-vous ces extases sublimes Que vous nous ravissez?

ô lac ! rochers muets ! grottes ! forêt obscure !

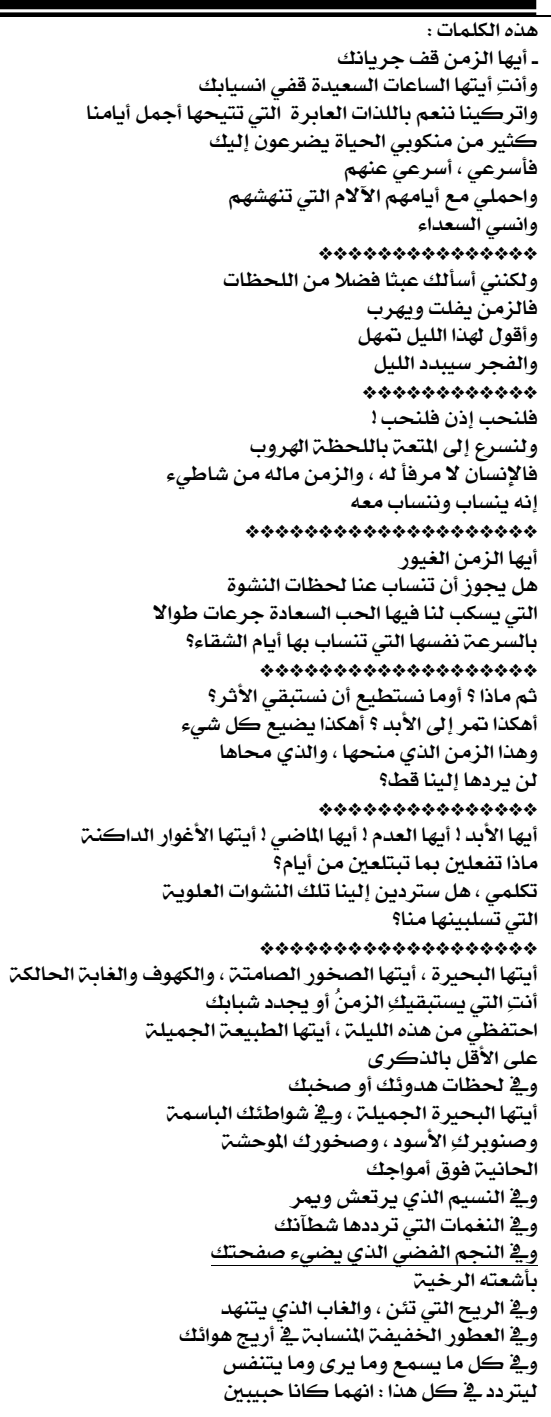

उo 
Vous, que le temps épargne ou qu'il peut rajeunir, Gardez de cette nuit, gardez, belle nature, Au moins le souvenir !

Qu'il soit dans ton repos, qu'il soit dans tes orages, Beau lac, et dans l'aspect de tes riants coteaux, Et dans ces noirs sapins, et dans ces rocs sauvages Qui pendent sur tes eaux.

Qu'il soit dans le zéphyr qui frémit et qui passe, Dans les bruits de tes bords par tes bords répétés, Dans l'astre au front d'argent qui blanchit ta surface

De ses molles clartés.

Que le vent qui gémit, le roseau qui soupire, Que les parfums légers de ton air embaumé, Que tout ce qu'on entend, l'on voit ou l'on respire, Tout dise : Ils ont aimé !

\section{La traduction de professeur Idris El-Waghiche ${ }^{\text {th }}$}

Ainsi, toujours poussés vers de nouveaux rivages, Dans la nuit éternelle emportés sans retour, Ne pourrons-nous jamais sur l'océan des âges Jeter l'ancre un seul jour?

ô lac ! l'année à peine a fini sa carrière, Et près des flots chéris qu'elle devait revoir, Regarde ! je viens seul m'asseoir sur cette pierre Où tu la vis s'asseoir !

Tu mugissais ainsi sous ces roches profondes, Ainsi tu te brisais sur leurs flancs déchirés, Ainsi le vent jetait l'écume de tes ondes Sur ses pieds adorés.

Un soir, t'en souvient-il ? nous voguions en silence ; On n'entendait au loin, sur l'onde et sous les cieux, Que le bruit des rameurs qui frappaient en cadence Tes flots harmonieux.

Tout à coup des accents inconnus à la terre Du rivage charmé frappèrent les échos ; Le flot fut attentif, et la voix qui m'est chère Laissa tomber ces mots :

" Ô temps ! suspends ton vol, et vous, heures propices ! Suspendez votre cours :
هكذا... نحن مدفوعون دوما إلى عواله جديدة

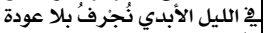

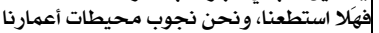
تَرُك المرساة ليوم يوم واحدى نجئ آهـ يا بحيرة ! العامُ قد أنهى بالكِاد دَوْرَته

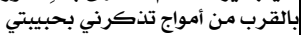

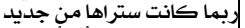
انظري....ها أنا قد عُدراتُ

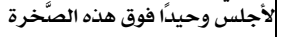

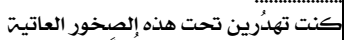

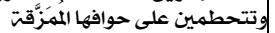

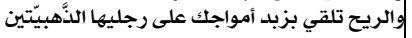
ذات مساء، إن كنت تذكرين؟ كنا نُبحرُ ِِِِ صَمَت

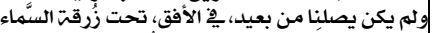

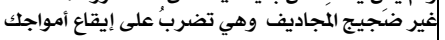

وفجأة لهجات غير مألوفت بِ عالمنا

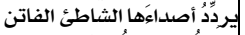

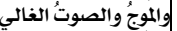

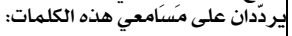

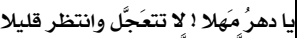

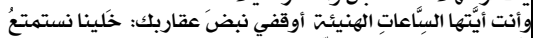

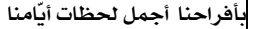

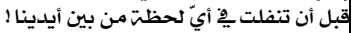

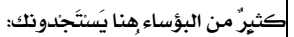

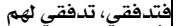

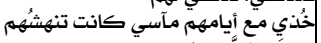

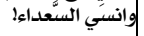

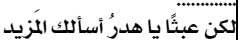

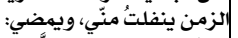

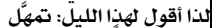

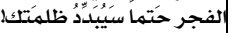
نَعْشقُ إذن، و نحبِّ أكثر

$$
\text { "^آ) شاعر وكاتب مغربي - استاذ بجامعت سيدي محمد ين عبدا لله - فاس - المغرب }
$$


Laissez-nous savourer les rapides délices

Des plus beaux de nos jours !

" Assez de malheureux ici-bas vous implorent, Coulez, coulez pour eux ;

Prenez avec leurs jours les soins qui les dévorent ;

Oubliez les heureux.

" Mais je demande en vain quelques moments encore, Le temps m'échappe et fuit ;

Je dis à cette nuit : Sois plus lente; et l'aurore

Va dissiper la nuit.

" Aimons donc, aimons donc ! de l'heure fugitive, Hâtons-nous, jouissons !

L'homme n'a point de port, le temps n'a point de rive ; Il coule, et nous passons ! "

Temps jaloux, se peut-il que ces moments d'ivresse, Où l'amour à longs flots nous verse le bonheur, S'envolent loin de nous de la même vitesse Que les jours de malheur?

Eh quoi ! n'en pourrons-nous fixer au moins la trace? Quoi ! passés pour jamais ! quoi ! tout entiers perdus ! Ce temps qui les donna, ce temps qui les efface, Ne nous les rendra plus !

Éternité, néant, passé, sombres abîmes, Que faites-vous des jours que vous engloutissez ? Parlez : nous rendrez-vous ces extases sublimes Que vous nous ravissez?

Ô lac ! rochers muets ! grottes ! forêt obscure ! Vous, que le temps épargne ou qu'il peut rajeunir, Gardez de cette nuit, gardez, belle nature,

Au moins le souvenir !

Qu'il soit dans ton repos, qu'il soit dans tes orages, Beau lac, et dans l'aspect de tes riants coteaux, Et dans ces noirs sapins, et dans ces rocs sauvages Qui pendent sur tes eaux.

Qu'il soit dans le zéphyr qui frémit et qui passe, Dans les bruits de tes bords par tes bords répétés, Dans l'astre au front d'argent qui blanchit ta surface De ses molles clartés.

Que le vent qui gémit, le roseau qui soupire, Que les parfums légers de ton air embaumé, Que tout ce qu'on entend, l'on voit ou l'on respire, Tout dise : Ils ont aimé !

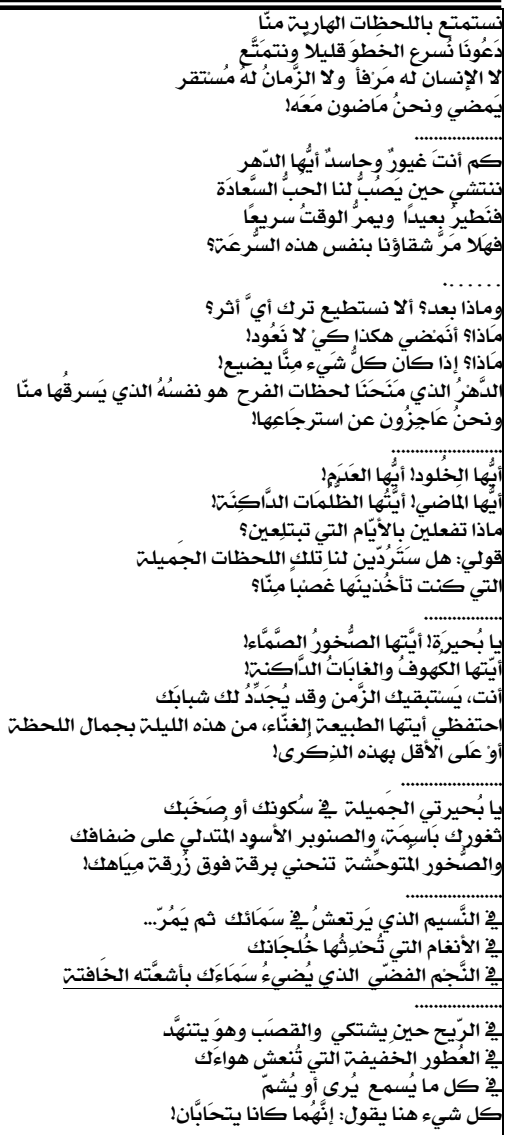

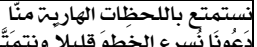

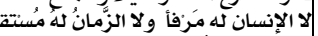

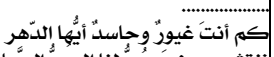

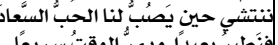

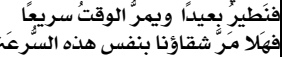
وماذا بعدى ألا نستطيع ترك أيَّ أثرى

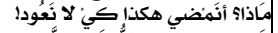

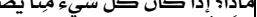

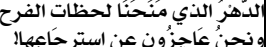

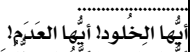

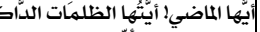

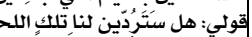

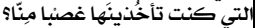

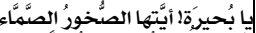

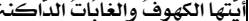

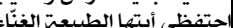
أو'

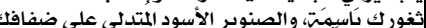

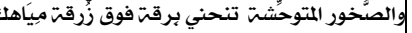

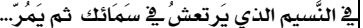

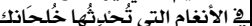

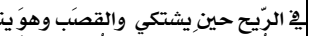

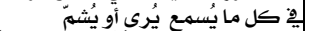
كل شيء هنا يقول: إنَّهما كانا يتحَابًان उठ 


\section{La traduction de Mohamed El-Samahy ${ }^{\text {19 }}$}

Ainsi, toujours poussés vers de nouveaux rivages, Dans la nuit éternelle emportés sans retour, Ne pourrons-nous jamais sur l'océan des âges Jeter l'ancre un seul jour?

Ô lac ! l'année à peine a fini sa carrière, Et près des flots chéris qu'elle devait revoir, Regarde! je viens seul m'asseoir sur cette pierre Où tu la vis s'asseoir !

Tu mugissais ainsi sous ces roches profondes, Ainsi tu te brisais sur leurs flancs déchirés, Ainsi le vent jetait l'écume de tes ondes Sur ses pieds adorés.

Un soir, t'en souvient-il ? nous voguions en silence ;

On n'entendait au loin, sur l'onde et sous les cieux,

Que le bruit des rameurs qui frappaient en cadence

Tes flots harmonieux.

Tout à coup des accents inconnus à la terre

Du rivage charmé frappèrent les échos ;

Le flot fut attentif, et la voix qui m'est chère

Laissa tomber ces mots :

" Ô temps ! suspends ton vol, et vous, heures propices!

Suspendez votre cours :

Laissez-nous savourer les rapides délices

Des plus beaux de nos jours !

" Assez de malheureux ici-bas vous implorent, Coulez, coulez pour eux ;

Prenez avec leurs jours les soins qui les dévorent ; Oubliez les heureux.

" Mais je demande en vain quelques moments encore,

Le temps m'échappe et fuit ;

Je dis à cette nuit : Sois plus lente; et l'aurore Va dissiper la nuit.

" Aimons donc, aimons donc ! de l'heure fugitive, Hâtons-nous, jouissons !

L'homme n'a point de port, le temps n'a point de rive ;

Il coule, et nous passons ! "

Temps jaloux, se peut-il que ces moments d'ivresse,

Où l'amour à longs flots nous verse le bonheur,

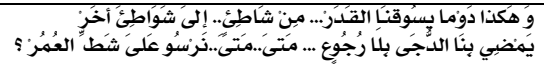
*****

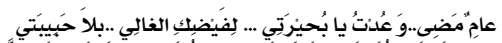

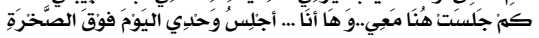
****

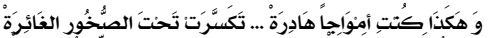

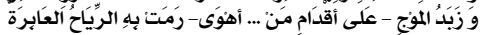

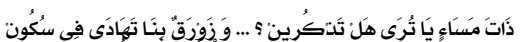

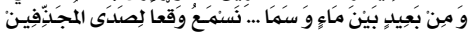
*****

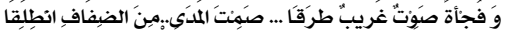

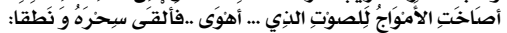
****

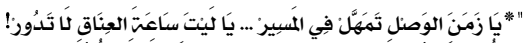

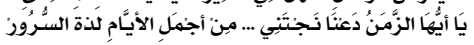
****

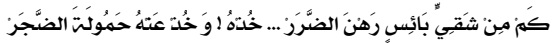

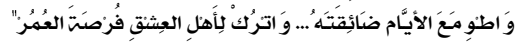
******

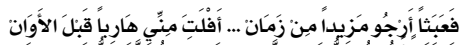

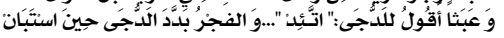
****

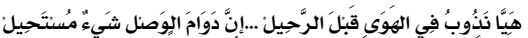

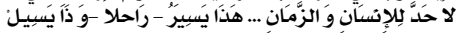

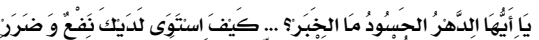

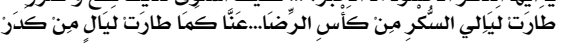
****

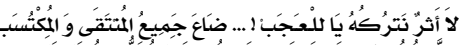

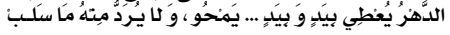
****

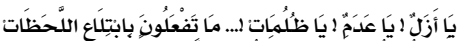

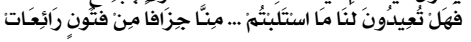
****

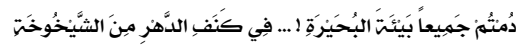

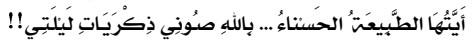
****

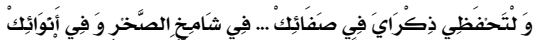

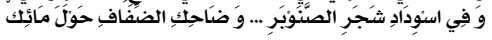
*****

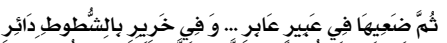

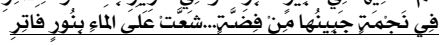
****

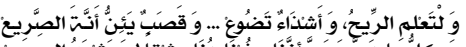

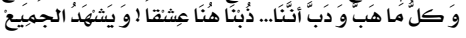


S'envolent loin de nous de la même vitesse

Que les jours de malheur?

Eh quoi ! n'en pourrons-nous fixer au moins la trace?

Quoi ! passés pour jamais ! quoi ! tout entiers perdus !

Ce temps qui les donna, ce temps qui les efface, Ne nous les rendra plus !

Éternité, néant, passé, sombres abîmes, Que faites-vous des jours que vous engloutissez ? Parlez : nous rendrez-vous ces extases sublimes Que vous nous ravissez? Ô lac ! rochers muets ! grottes ! forêt obscure ! Vous, que le temps épargne ou qu'il peut rajeunir, Gardez de cette nuit, gardez, belle nature,

Au moins le souvenir !

Qu'il soit dans ton repos, qu'il soit dans tes orages, Beau lac, et dans l'aspect de tes riants coteaux, Et dans ces noirs sapins, et dans ces rocs sauvages Qui pendent sur tes eaux.

Qu'il soit dans le zéphyr qui frémit et qui passe, Dans les bruits de tes bords par tes bords répétés, Dans l'astre au front d'argent qui blanchit ta surface

De ses molles clartés.

Que le vent qui gémit, le roseau qui soupire, Que les parfums légers de ton air embaumé, Que tout ce qu'on entend, l'on voit ou l'on respire, Tout dise : Ils ont aimé ! 


\section{ملخص البحث}

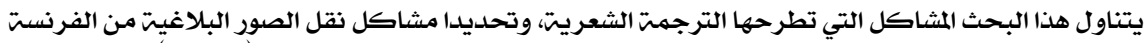

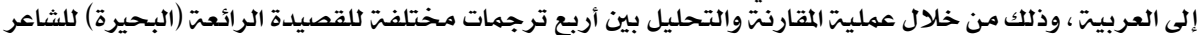

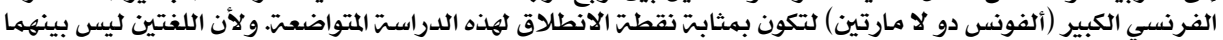

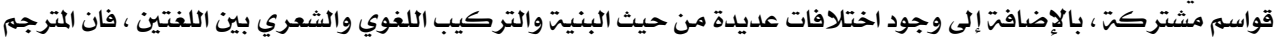

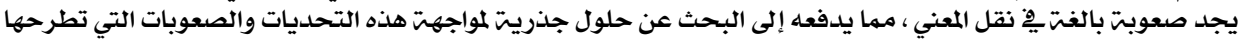
الترجمت الشعريت.

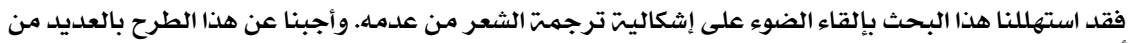

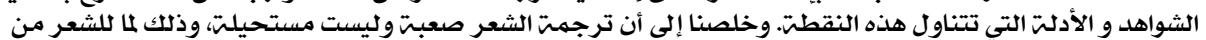

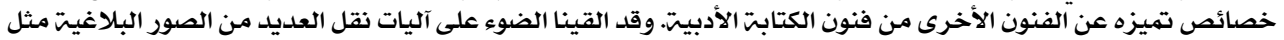

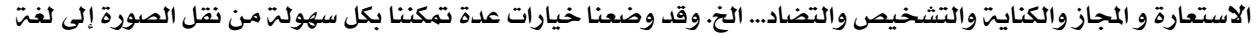

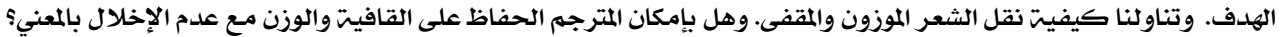

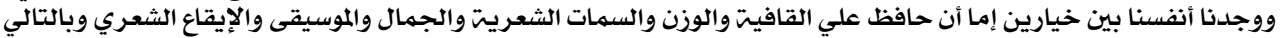

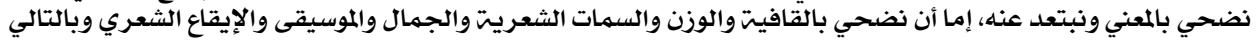

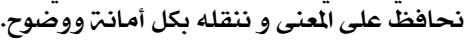

\section{Abstract}

This research deals with the problems posed by poetic translation, in particular the problems of transferring rhetorical images from French to Arabic. Through the process of comparison and analysis between four different translations of the magnificent poem (lake) of the great french poet (Alphonse de Lamartine) to serve as the starting point for this modest study. As both languages have no common denominators, in addition to the existence of many differences in terms of structure and syntax linguistic and poetic between the two languages, the translator finds it very difficult to convey the meaning, which leads to the search for radical solutions to meet these challenges and difficulties posed by the translation of poetry.

We have started this research by shedding light on the problem of translating poetry. We have responded to this proposition with much evidence that addresses this point. We concluded that translation of poetry is difficult and not impossible, because of the characteristics of poetry is distinguished from other arts of literary writing. We have highlighted the mechanisms of transferring many rhetorical images such as metaphor, 
synecdoche, periphrasis, diagnosis, antagonism, etc. We have developed several options that enable us to easily transfer the image to the target language. We discussed how to transfer rhymed poetry. 